\title{
Supporting Information: A residual entropy scaling approach for viscosity based on the GERG-2008 equation of state
}

\author{
Jonas Mairhofer* \\ BASF SE, 67056 Ludwigshafen, Germany \\ E-mail: jonas.mairhofer@basf.com
}




\section{Optimized pure-component parameters}

Table S1 presents the optimized parameters for every compound to be used in eq. 4 of the main manuscript in order to correlate the scaled pure-component viscosity with residual entropy.

Table S1: Values for the optimized coefficients of eq. 4 of the main manuscript for the compounds studied in this work. For compounds labeled with a star, the value of $D$ is determined from the correlation given by eq. 6 of the main manuscript.

\begin{tabular}{l|c|c|c|c} 
& $A$ & $B$ & $C$ & $D$ \\
\hline methane & $-3.14160 \mathrm{E}-02$ & $-7.22738 \mathrm{E}-01$ & $1.13859 \mathrm{E}-01$ & $1.16905 \mathrm{E}-02$ \\
ethane & $-1.87015 \mathrm{E}-01$ & $-9.98938 \mathrm{E}-01$ & $-4.45252 \mathrm{E}-02$ & $-3.07766 \mathrm{E}-03$ \\
propane & $-2.38359 \mathrm{E}-01$ & $-9.72242 \mathrm{E}-01$ & $-4.71452 \mathrm{E}-02$ & $-3.18179 \mathrm{E}-03$ \\
n-butane & $-2.05100 \mathrm{E}-01$ & $-8.31992 \mathrm{E}-01$ & $-1.48650 \mathrm{E}-02$ & $-5.46152 \mathrm{E}-04$ \\
isobutane & $-1.70080 \mathrm{E}-01$ & $-8.31823 \mathrm{E}-01$ & $-1.10934 \mathrm{E}-02$ & $-1.09953 \mathrm{E}-03$ \\
n-pentane* & $-2.31712 \mathrm{E}-01$ & $-9.08403 \mathrm{E}-01$ & $-4.81325 \mathrm{E}-02$ & $-2.26804 \mathrm{E}-03$ \\
isopentane & $-1.42415 \mathrm{E}-01$ & $-8.88522 \mathrm{E}-01$ & $-4.56753 \mathrm{E}-02$ & $-3.21314 \mathrm{E}-03$ \\
hexane & $-2.79791 \mathrm{E}-01$ & $-8.84493 \mathrm{E}-01$ & $-5.20562 \mathrm{E}-02$ & $-3.13857 \mathrm{E}-03$ \\
heptane & $-2.47853 \mathrm{E}-01$ & $-8.41157 \mathrm{E}-01$ & $-4.54250 \mathrm{E}-02$ & $-2.21548 \mathrm{E}-03$ \\
octane & $-4.98731 \mathrm{E}-01$ & $-9.09925 \mathrm{E}-01$ & $-5.66863 \mathrm{E}-02$ & $-2.66169 \mathrm{E}-03$ \\
nonane & $-4.38367 \mathrm{E}-01$ & $-9.11909 \mathrm{E}-01$ & $-6.50389 \mathrm{E}-02$ & $-3.26398 \mathrm{E}-03$ \\
decane & $-1.30465 \mathrm{E}-01$ & $-7.29921 \mathrm{E}-01$ & $-3.77324 \mathrm{E}-02$ & $-1.78622 \mathrm{E}-03$ \\
$\mathrm{CO}^{*}$ & $-8.82515 \mathrm{E}-03$ & $-6.53553 \mathrm{E}-01$ & $7.10728 \mathrm{E}-02$ & $1.06831 \mathrm{E}-03$ \\
$\mathrm{CO}_{2}$ & $-2.63706 \mathrm{E}-01$ & $-8.82637 \mathrm{E}-01$ & $-1.19515 \mathrm{E}-02$ & $2.21305 \mathrm{E}-03$ \\
$\mathrm{H}_{2}{ }^{*}$ & $1.25418 \mathrm{E}-01$ & $-5.08704 \mathrm{E}-01$ & $8.12269 \mathrm{E}-01$ & $2.04251 \mathrm{E}-02$ \\
$\mathrm{O}_{2}{ }^{*}$ & $-1.21504 \mathrm{E}-01$ & $-9.52554 \mathrm{E}-01$ & $3.72548 \mathrm{E}-02$ & $8.72080 \mathrm{E}-03$ \\
$\mathrm{~N}_{2}{ }^{*}$ & $-8.08926 \mathrm{E}-02$ & $-9.03072 \mathrm{E}-01$ & $2.96500 \mathrm{E}-02$ & $1.94214 \mathrm{E}-03$ \\
$\mathrm{H}_{2} \mathrm{~S}$ & $-5.87760 \mathrm{E}-02$ & $-8.44317 \mathrm{E}-01$ & $-1.35260 \mathrm{E}-02$ & $-3.72996 \mathrm{E}-03$ \\
$\mathrm{H}_{2} \mathrm{O}$ & $4.17549 \mathrm{E}-02$ & $-4.34449 \mathrm{E}-01$ & $-1.57058 \mathrm{E}-02$ & $-6.22529 \mathrm{E}-03$ \\
helium $^{*}$ & $2.54306 \mathrm{E}-01$ & $-1.44743 \mathrm{E}-01$ & $8.90411 \mathrm{E}-01$ & $2.03250 \mathrm{E}-03$ \\
argon $^{*}$ & $-6.55869 \mathrm{E}-02$ & $-9.61670 \mathrm{E}-01$ & $4.85341 \mathrm{E}-02$ & $7.28174 \mathrm{E}-03$
\end{tabular}




\section{Range of residual entropy used for parameter adjust-}

\section{ment}

Table S2 presents for all 21 components studied in this work the range of residual entropy used for adjusting the coefficients for eq. 4 of the main manuscript. Furthermore, if the polynomial of a given compound exhibits a maximum for $\ln \eta *$ at a value of residual entropy $s^{r e s} / \mathrm{R}>-15$, the location of this maximum is given.

Table S2: Minimal and maximal value of residual entropy used in the adjustment of the coefficients for eq. 4 in the main manuscript. Furthermore, the location of the maximum of the polynomial if it occurs at values of $s^{\text {res }} / \mathrm{R}>-15$.

\begin{tabular}{l|c|c|c} 
& $s_{\text {min }}^{\text {res }} / \mathrm{R}$ & $s_{\text {max }}^{\text {res }} / \mathrm{R}$ & $s^{\text {res }} / \mathrm{R}$ at max. \\
\hline methane & -4.099 & $-2.165 \mathrm{e}-3$ & -7.543 \\
ethane & -7.186 & $-9.779 \mathrm{e}-2$ & $<-15$ \\
propane & -10.308 & $-1.017 \mathrm{e}-2$ & $<-15$ \\
n-butane & -8.979 & $-1.381 \mathrm{e}-2$ & $<-15$ \\
isobutane & -9.756 & $-4.855 \mathrm{e}-3$ & $<-15$ \\
n-pentane & -5.804 & $-1.083 \mathrm{e}-2$ & $<-15$ \\
isopentane & -5.411 & $-9.618 \mathrm{e}-2$ & $<-15$ \\
hexane & -9.695 & -0.131 & $<-15$ \\
heptane & -8.454 & -0.222 & $<-15$ \\
octane & -8.982 & -0.225 & $<-15$ \\
nonane & -11.855 & -2.527 & $<-15$ \\
decane & -10.739 & $-1.132 \mathrm{e}-2$ & $<-15$ \\
$\mathrm{CO}$ & -4.449 & $-6.167 \mathrm{e}-4$ & $<-15$ \\
$\mathrm{CO}$ & -4.888 & $-9.779 \mathrm{e}-2$ & -9.219 \\
$\mathrm{H}_{2}$ & -1.897 & $-5.761 \mathrm{e}-4$ & $<-15$ \\
$\mathrm{O}_{2}$ & -4.235 & -0.118 & -7.624 \\
$\mathrm{~N}_{2}$ & -4.364 & $-1.468 \mathrm{e}-3$ & $<-15$ \\
$\mathrm{H}_{2} \mathrm{~S}$ & -4.939 & $-3.133 \mathrm{e}-4$ & $<-15$ \\
$\mathrm{H}_{2} \mathrm{O}$ & -7.657 & $-1.011 \mathrm{e}-2$ & $<-15$ \\
helium & -0.681 & $-4.211 \mathrm{e}-4$ & $<-15$ \\
argon & -3.567 & $-9.411 \mathrm{e}-4$ & -8.595 \\
& & &
\end{tabular}




\section{Composition of the studied natural gas mixtures}

Table S3 presents the composition of the natural gas mixtures studied in the main manuscript.

\section{Results for natural gas systems}

Table S4 presents the deviations of several viscosity models for the natural gas mixtures studied in the main manuscript. These values correspond to Fig. 3 in the main manuscript.

\section{Results for binary and ternary mixtures}

Figures S1 to S6 visualize the deviations of the mixtures presented in Tables 4 and 5 in the main manuscript as a function of dimensionless residual entropy $s^{\text {res }} / \mathrm{R}$ and temperature.
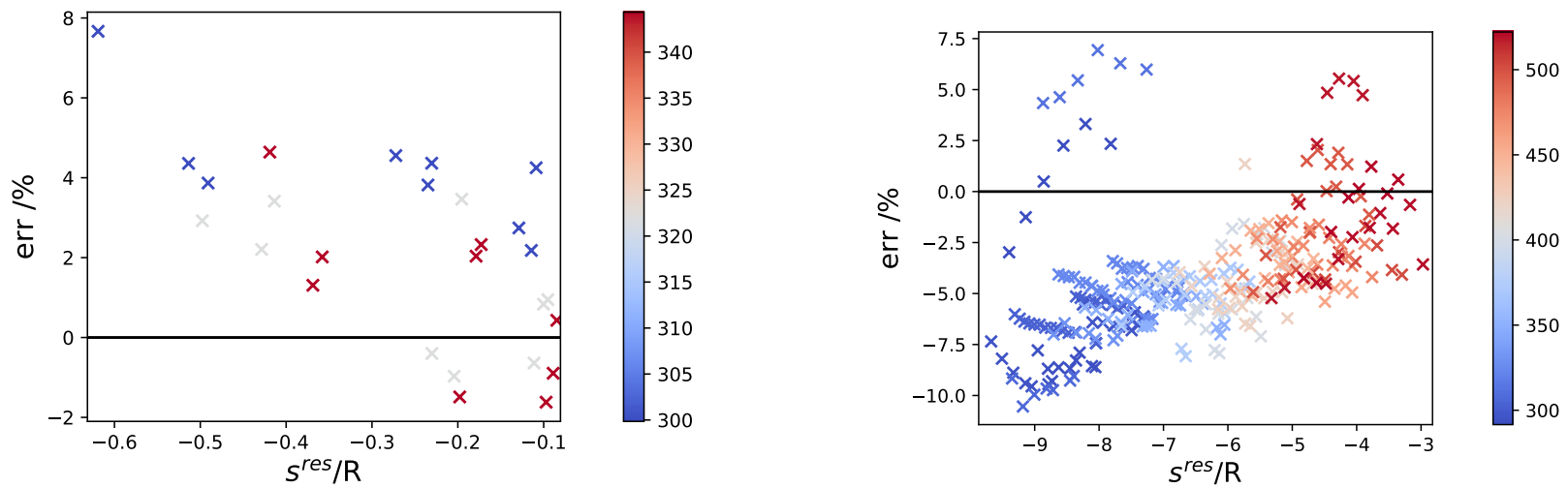

Figure S1: Deviations of the predicted viscosity as a function of dimensionless residual entropy $s^{\text {res }} / \mathrm{R}$ for the binary mixtures methane with $\mathrm{H}_{2} \mathrm{~S}$ (left) and heptane with decane (right). The color scale indicates temperature in Kelvin. 


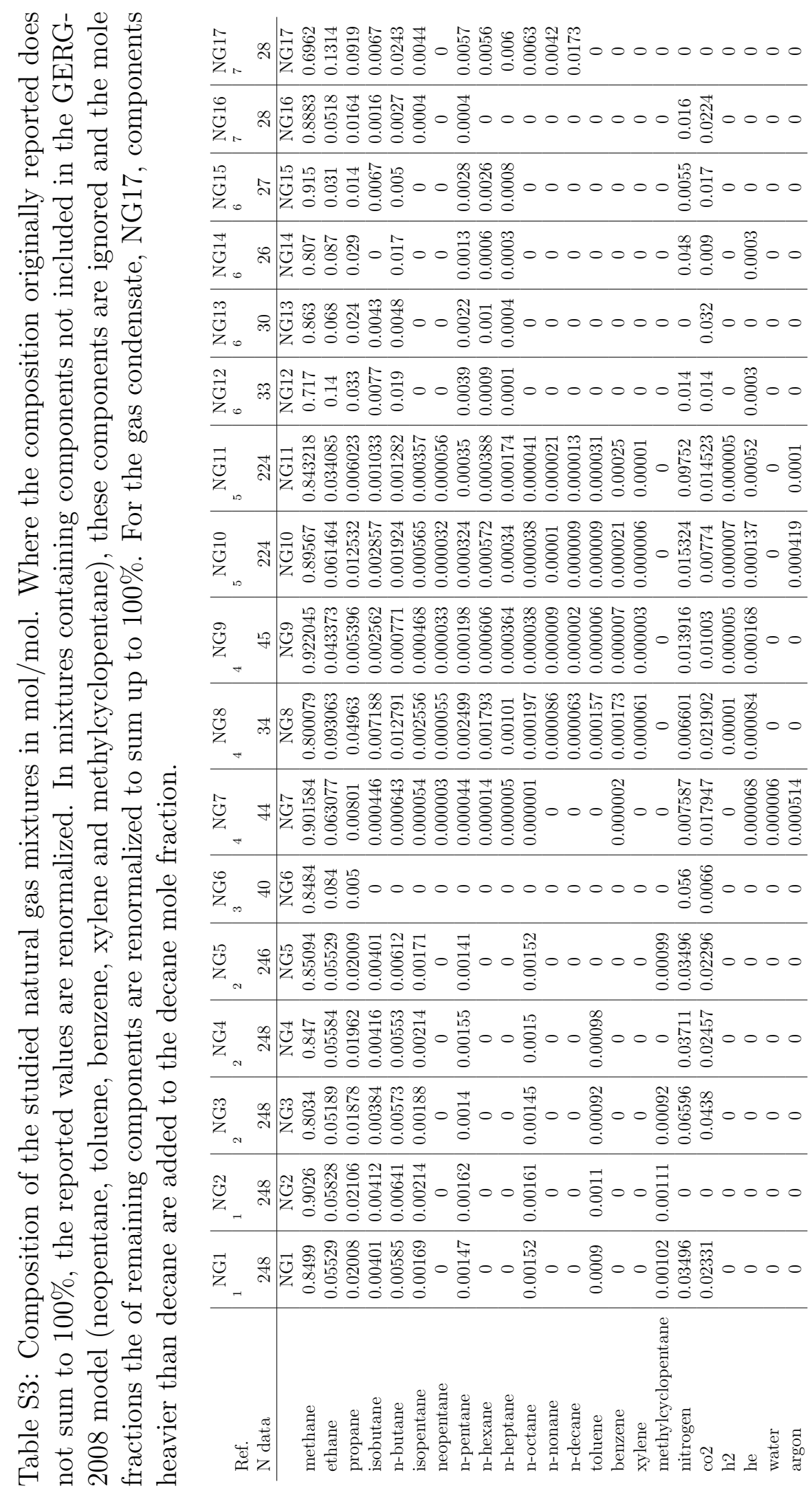




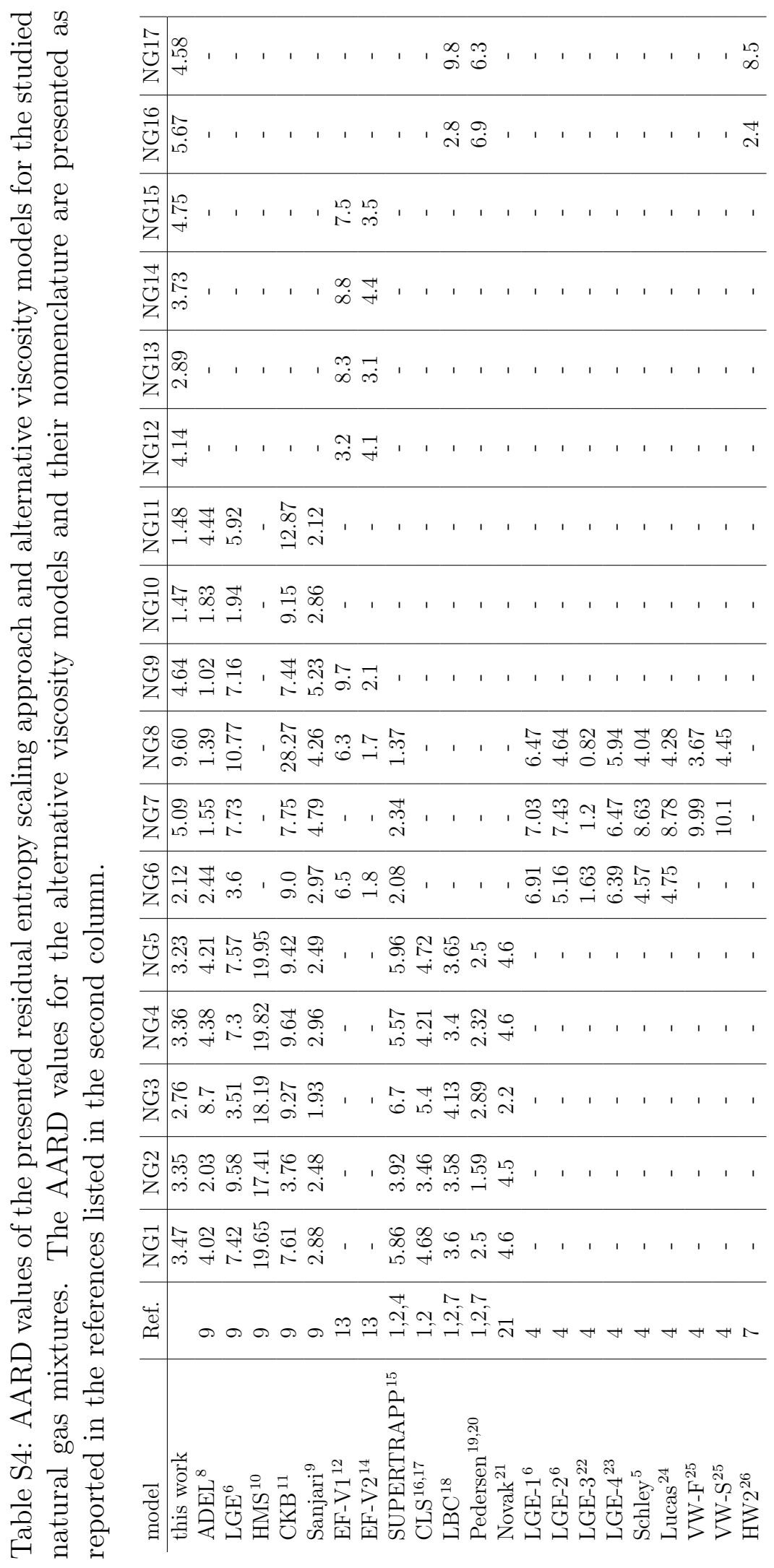



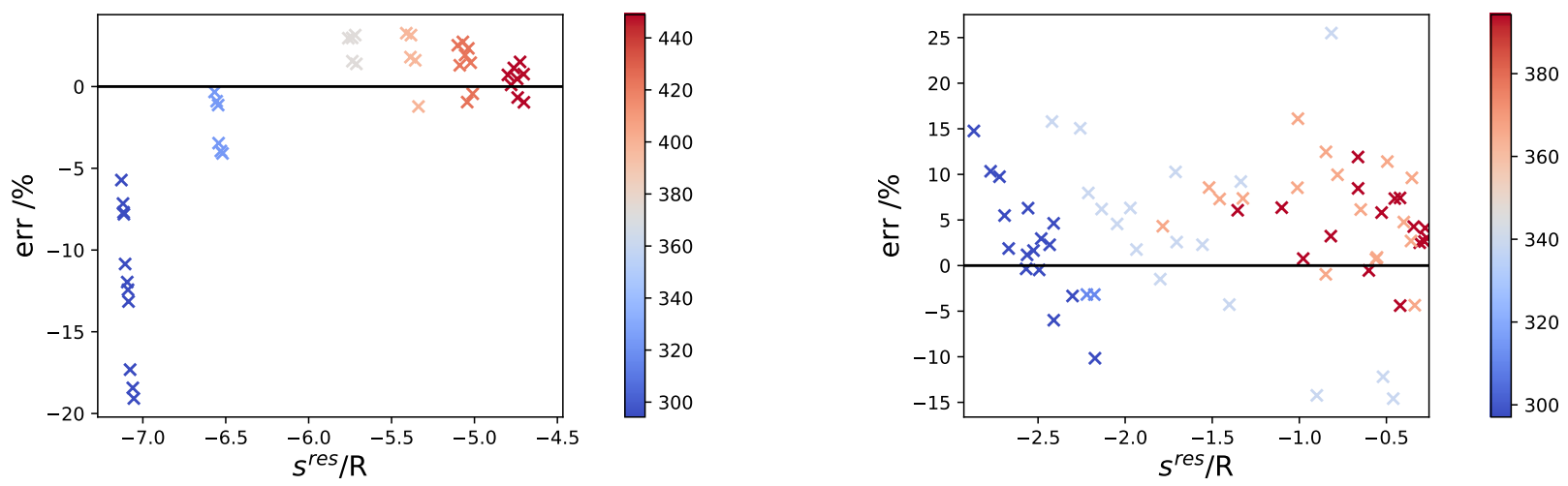

Figure S2: Deviations of the predicted viscosity as a function of dimensionless residual entropy $s^{\text {res }} / \mathrm{R}$ for the binary mixtures $\mathrm{CO}_{2}$ with $\mathrm{H}_{2} \mathrm{O}$ (left) and $\mathrm{CO}_{2}$ with $\mathrm{H}_{2} \mathrm{~S}$ (right). The color scale indicates temperature in Kelvin.
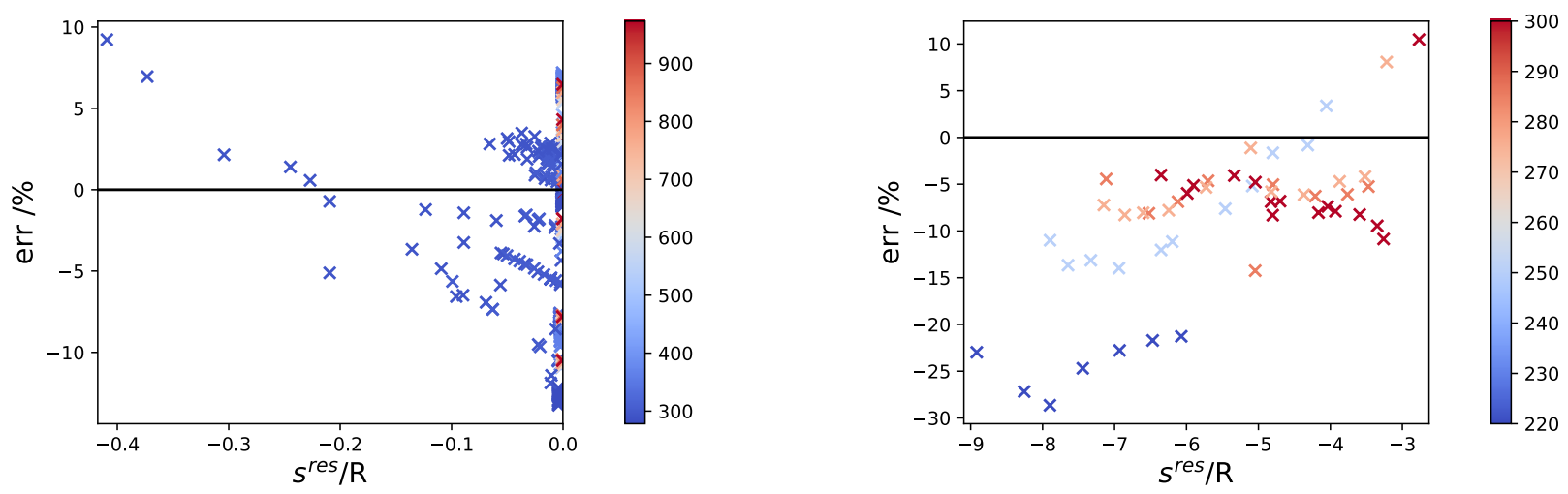

Figure S3: Deviations of the predicted viscosity as a function of dimensionless residual entropy $s^{r e s} / \mathrm{R}$ for the binary mixtures $\mathrm{CO}_{2}$ with helium (left) and $\mathrm{CO}_{2}$ with heptane (right). The color scale indicates temperature in Kelvin. 

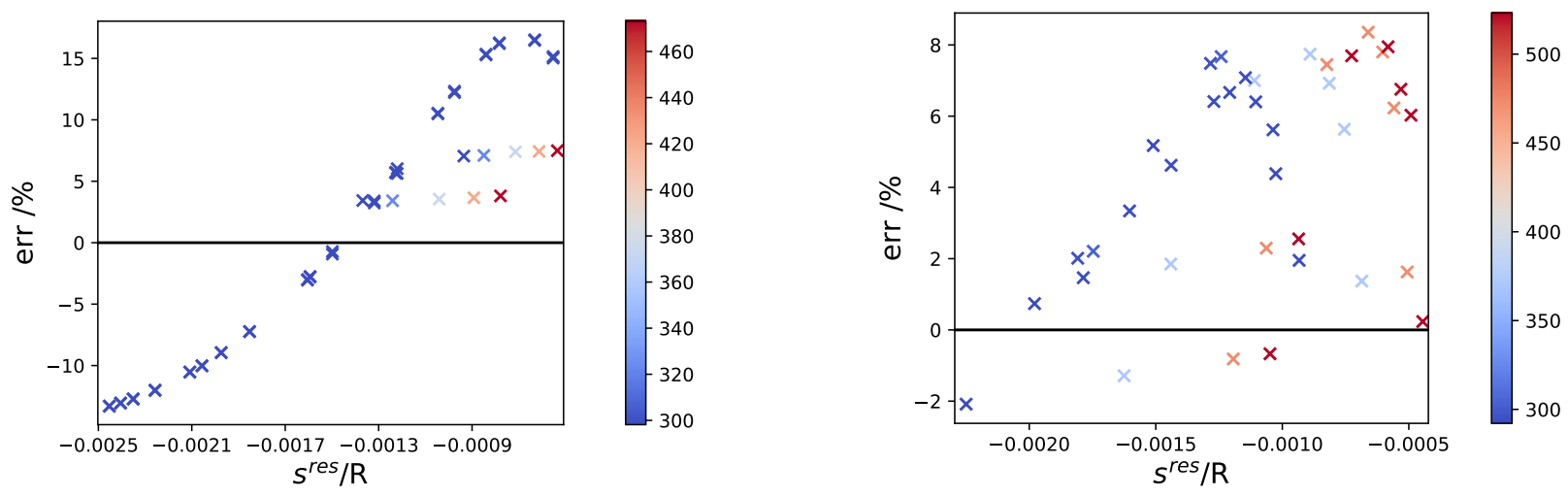

Figure S4: Deviations of the predicted viscosity as a function of dimensionless residual entropy $s^{r e s} / \mathrm{R}$ for the binary mixtures $\mathrm{CO}$ with helium (left) and $\mathrm{CO}$ with hydrogen (right). The color scale indicates temperature in Kelvin.
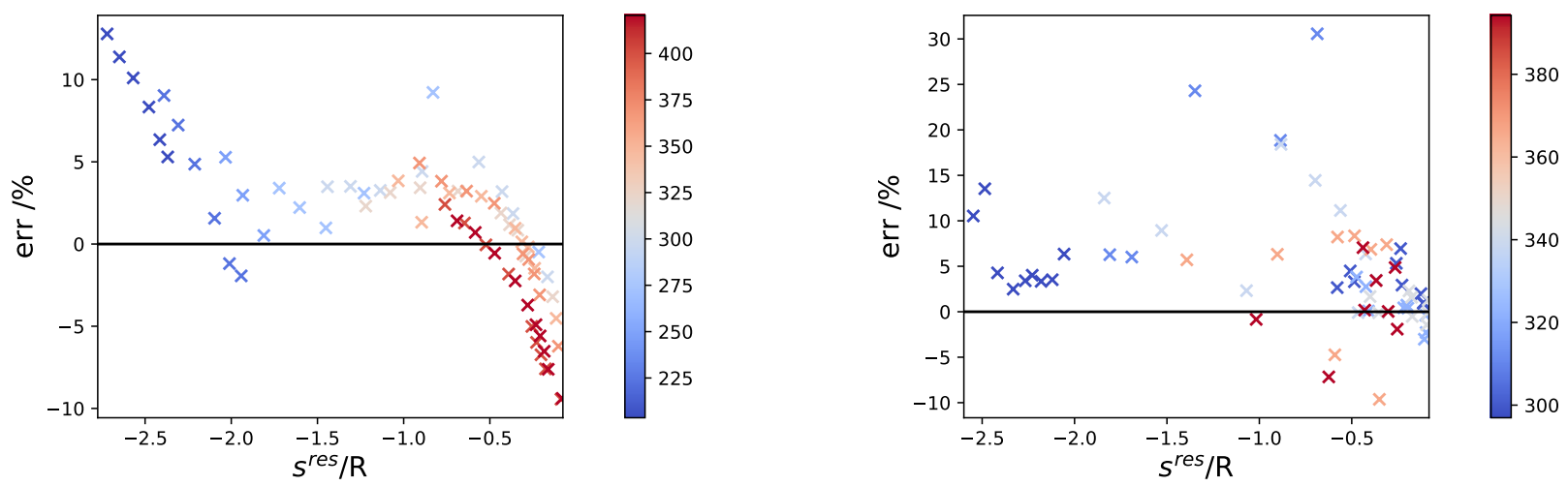

Figure S5: Deviations of the predicted viscosity as a function of dimensionless residual entropy $s^{r e s} / \mathrm{R}$ for the ternary mixtures methane with propane and $\mathrm{CO}_{2}$ (left) and methane with $\mathrm{CO}_{2}$ and $\mathrm{H}_{2} \mathrm{~S}$ (right). The color scale indicates temperature in Kelvin. 


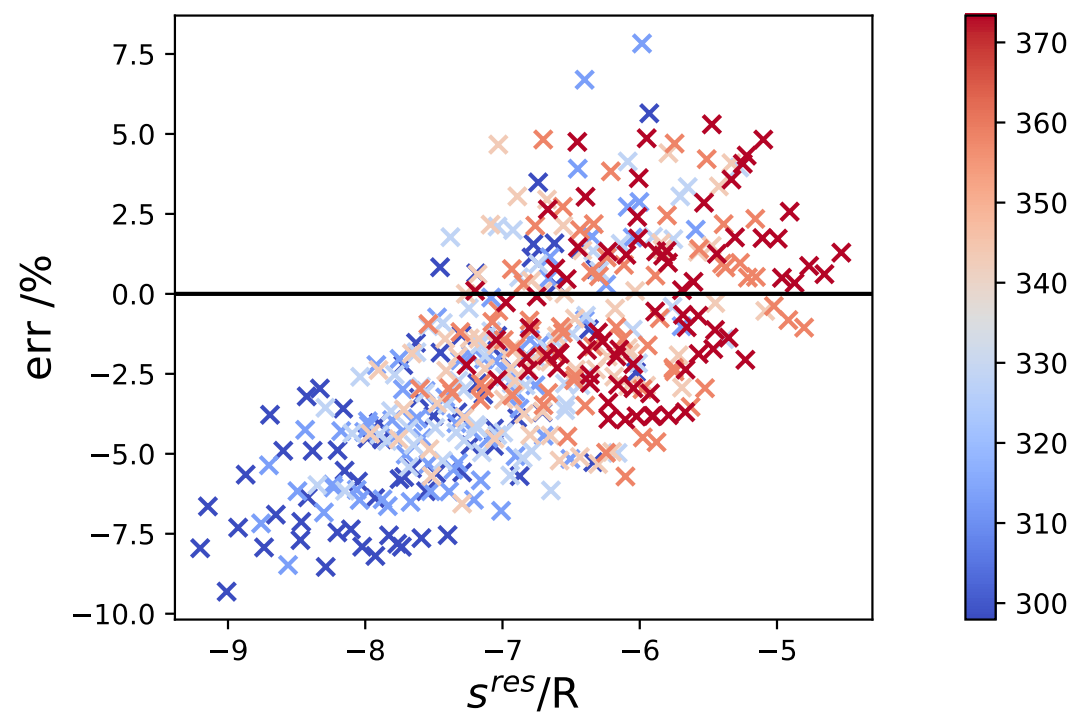

Figure S6: Deviations of the predicted viscosity as a function of dimensionless residual entropy $s^{\text {res }} / \mathrm{R}$ for the ternary mixture pentane with octane and decane. The color scale indicates temperature in Kelvin. 


\section{Evaluation of GERG-2008 residual entropies}

In order to evaluate the implementation of the GERG-2008 model and the obtained values for residual entropy $s^{\text {res }}$, pure-component values for $s^{\text {res }}$ are compared to results from another thermodynamic model, the perturbed-chain statistical association fluid theory (PCSAFT) ${ }^{27-31}$. As the following figures show, results of both thermodynamic models agree very well, confirming the correct implementation of the pure-component EOS of the GERG-2008 model and the reliability of results for residual entropy. Deviations in the vicinity of the critical point can be attributed to the deterioration of the performance of PC-SAFT at these conditions. In the case of $\mathrm{H}_{2}$, the PC-SAFT parameters lead to a clearly overestimated value for the critical temperature. For helium, no PC-SAFT parameters were available.
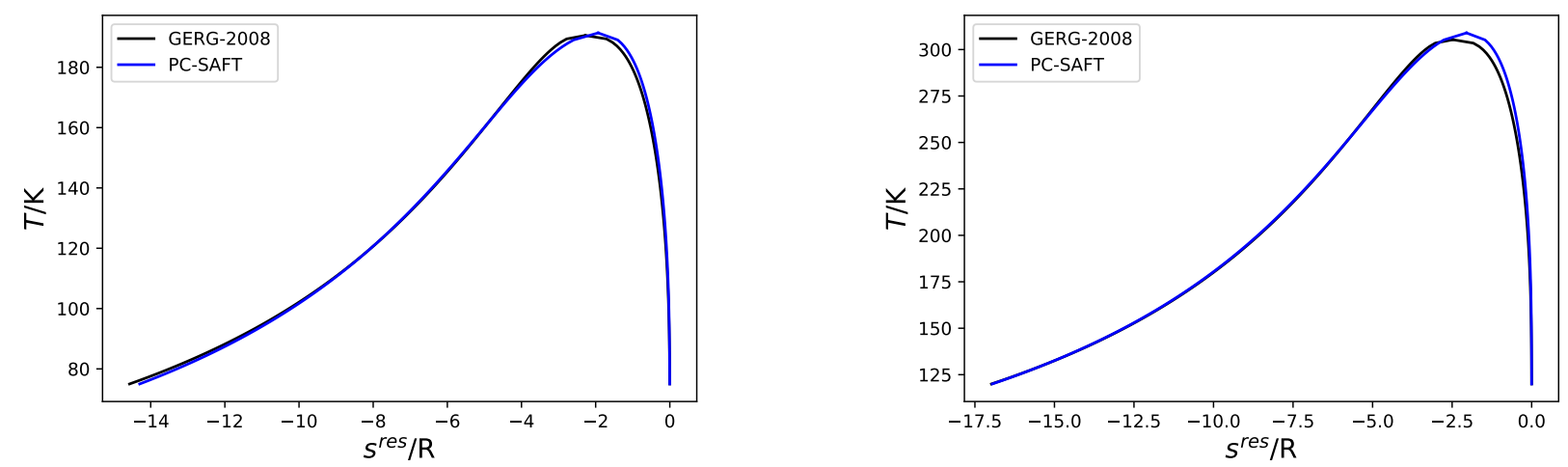

Figure S7: Residual entropy along the phase envelope for methane (left) and ethane (right) obtained from the pure-component EOS of GERG-2008 and PC-SAFT. PC-SAFT model parameters are taken from Ref. 27. 

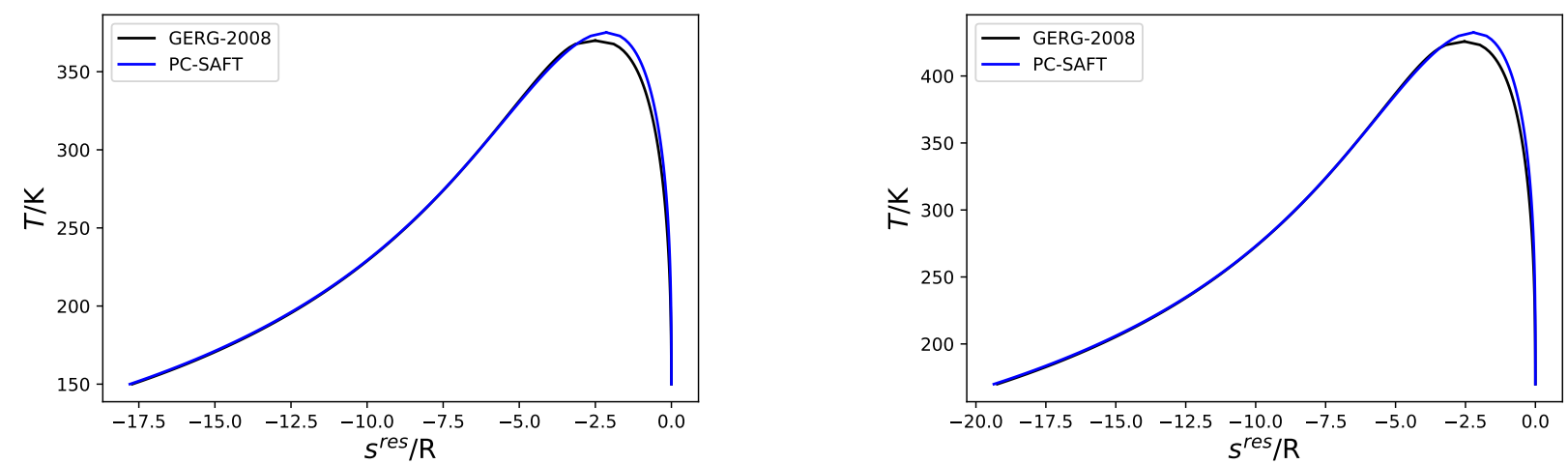

Figure S8: Residual entropy along the phase envelope for propane (left) and butane (right) obtained from the pure-component EOS of GERG-2008 and PC-SAFT. PC-SAFT model parameters are taken from Ref. 27.
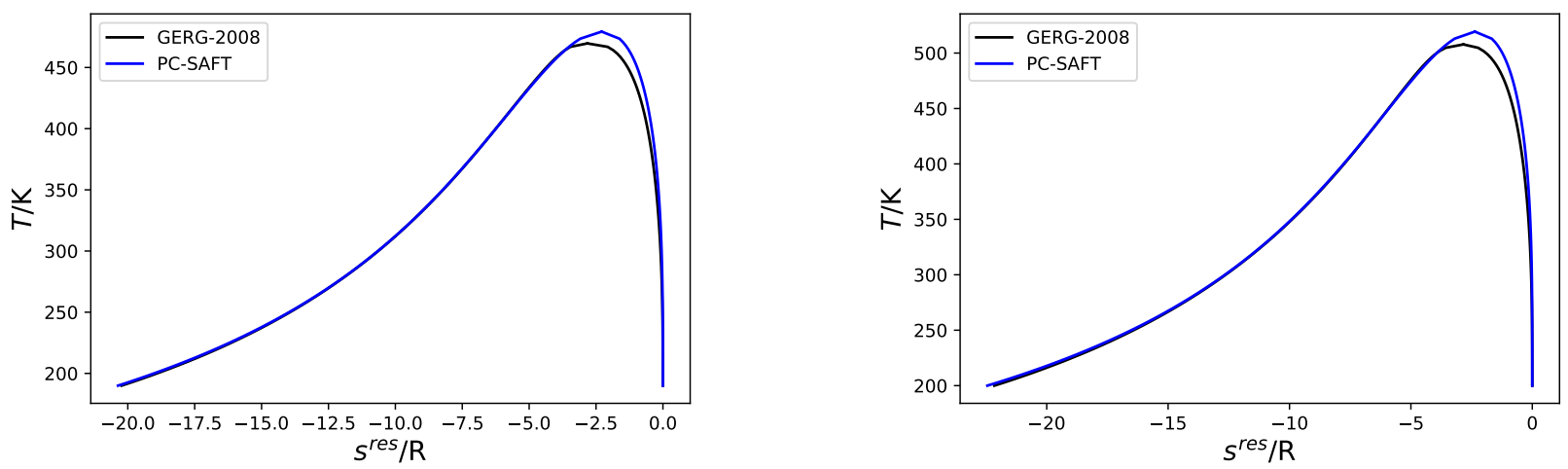

Figure S9: Residual entropy along the phase envelope for pentane (left) and hexane (right) obtained from the pure-component EOS of GERG-2008 and PC-SAFT. PC-SAFT model parameters are taken from Ref. 27.
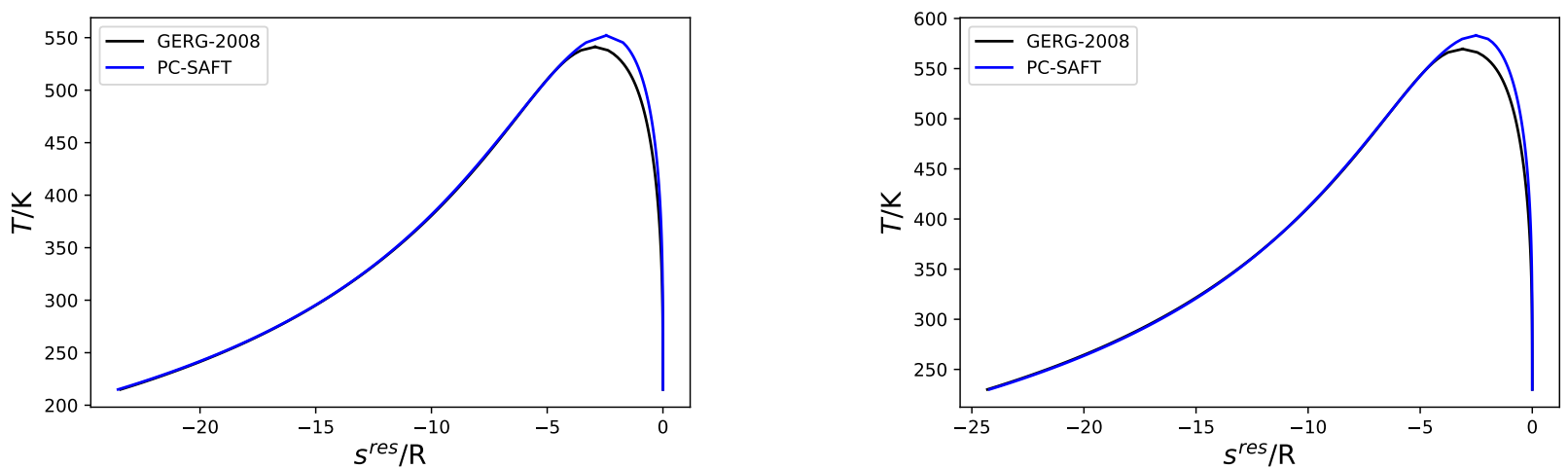

Figure S10: Residual entropy along the phase envelope for heptane (left) and octane (right) obtained from the pure-component EOS of GERG-2008 and PC-SAFT. PC-SAFT model parameters are taken from Ref. 27. 

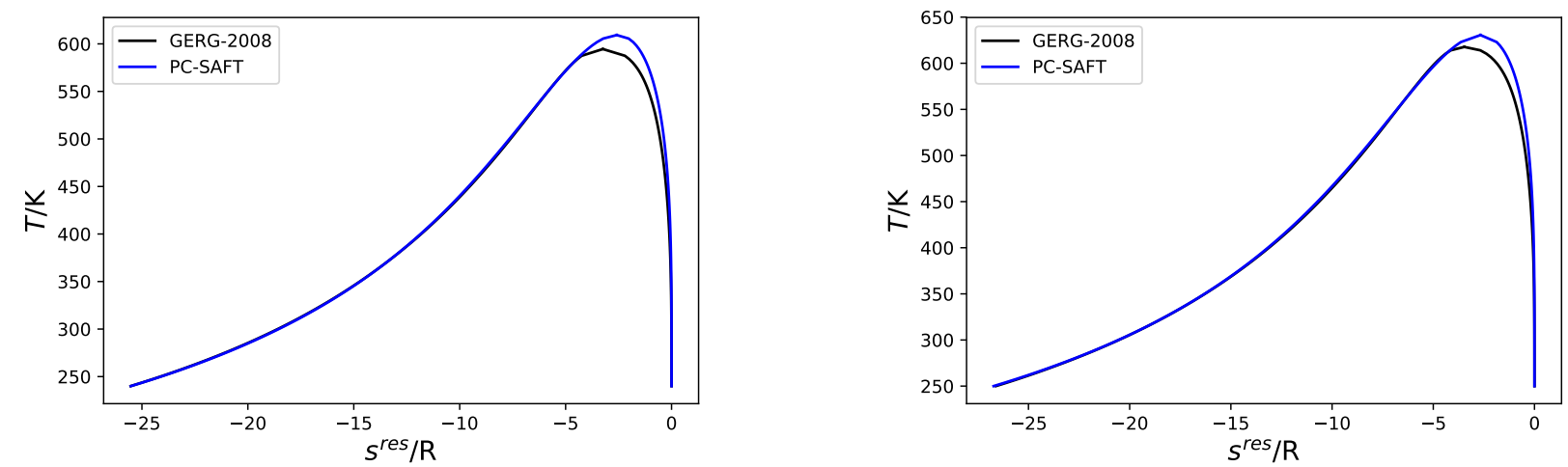

Figure S11: Residual entropy along the phase envelope for nonane (left) and decane (right) obtained from the pure-component EOS of GERG-2008 and PC-SAFT. PC-SAFT model parameters are taken from Ref. 27.
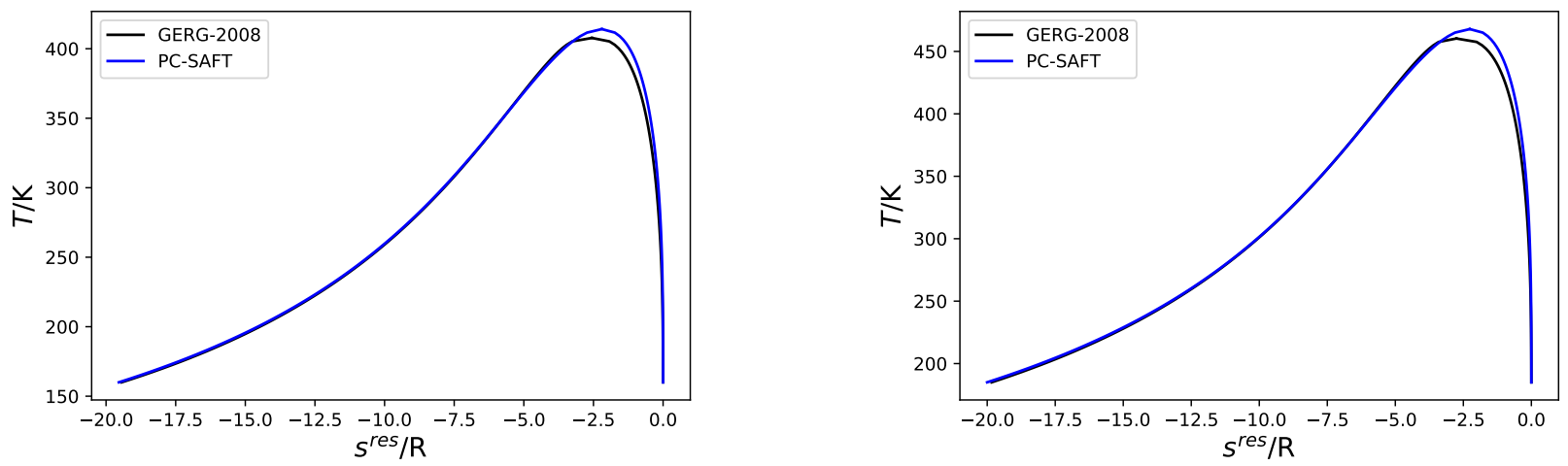

Figure S12: Residual entropy along the phase envelope for isobutane (left) and isopentane (right) obtained from the pure-component EOS of GERG-2008 and PC-SAFT. PC-SAFT model parameters are taken from Ref. 27.
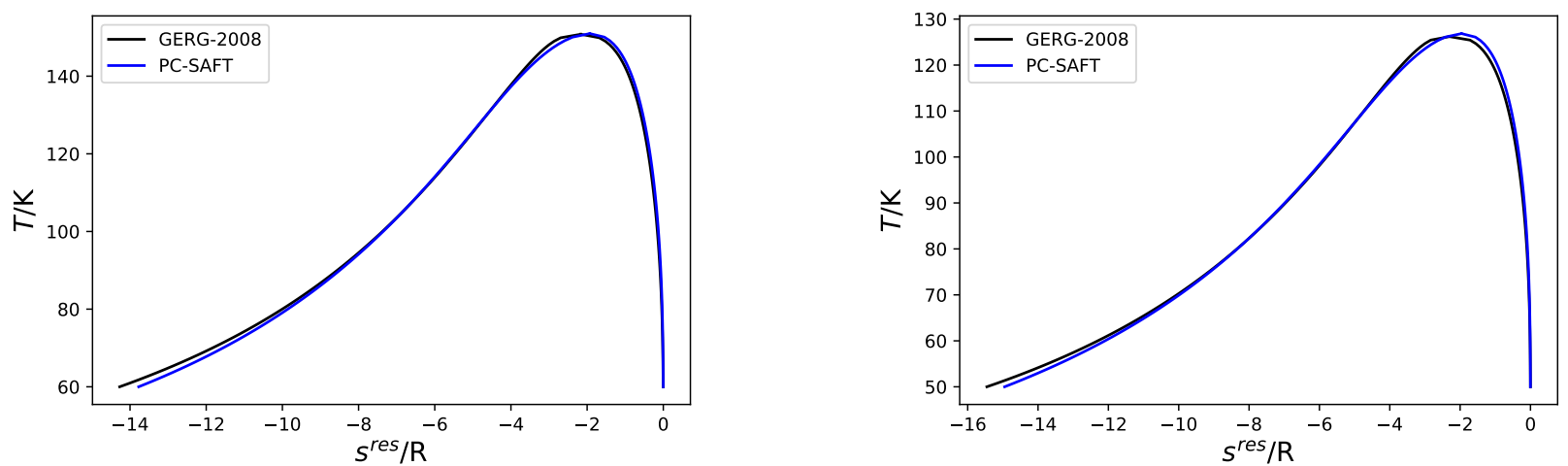

Figure S13: Residual entropy along the phase envelope for argon (left) and nitrogen (right) obtained from the pure-component EOS of GERG-2008 and PC-SAFT. PC-SAFT model parameters are taken from Refs. 27 and 29. 

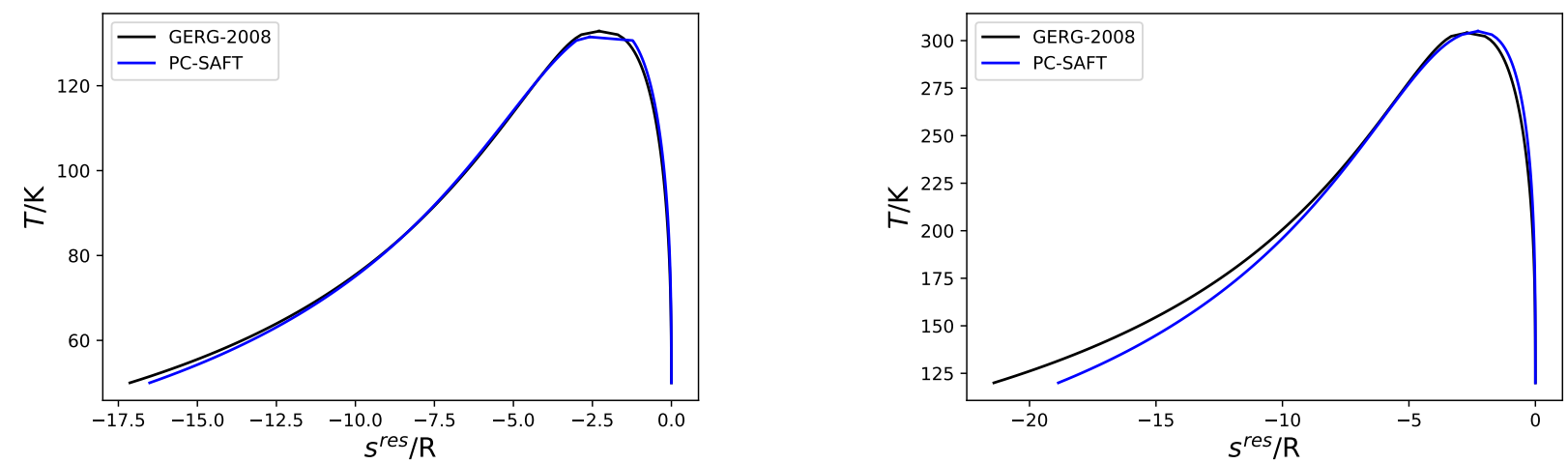

Figure S14: Residual entropy along the phase envelope for $\mathrm{CO}$ (left) and $\mathrm{CO}_{2}$ (right) obtained from the pure-component EOS of GERG-2008 and PC-SAFT. PC-SAFT model parameters are taken from Refs. 27 and 29.
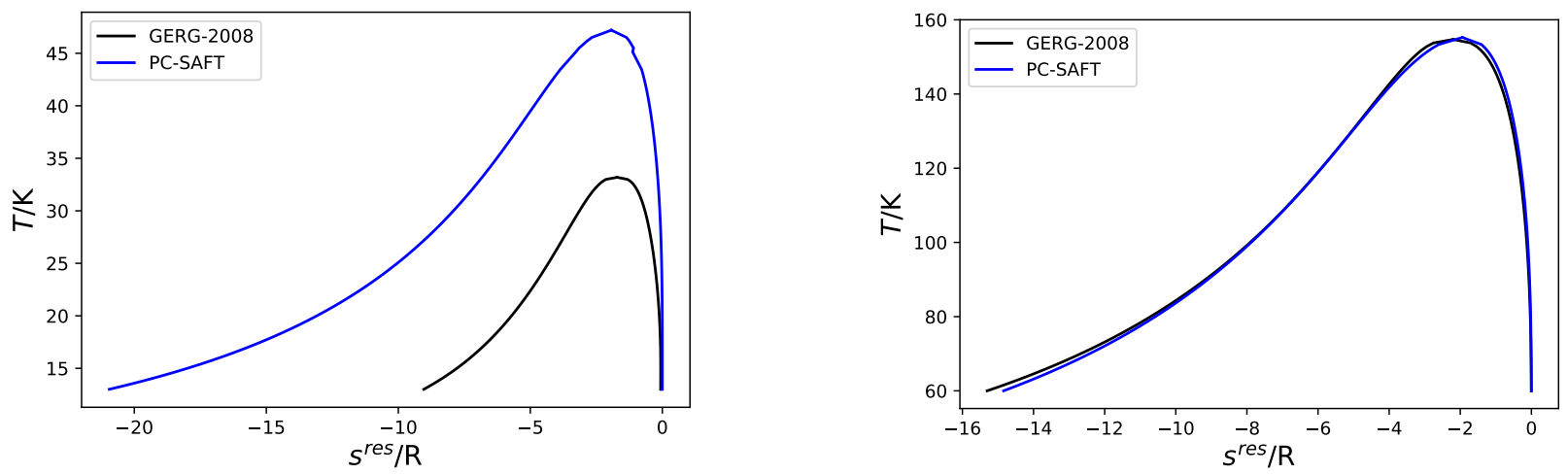

Figure S15: Residual entropy along the phase envelope for hydrogen (left) and oxygen (right) obtained from the pure-component EOS of GERG-2008 and PC-SAFT. PC-SAFT model parameters are taken from Refs. 32 and 33.
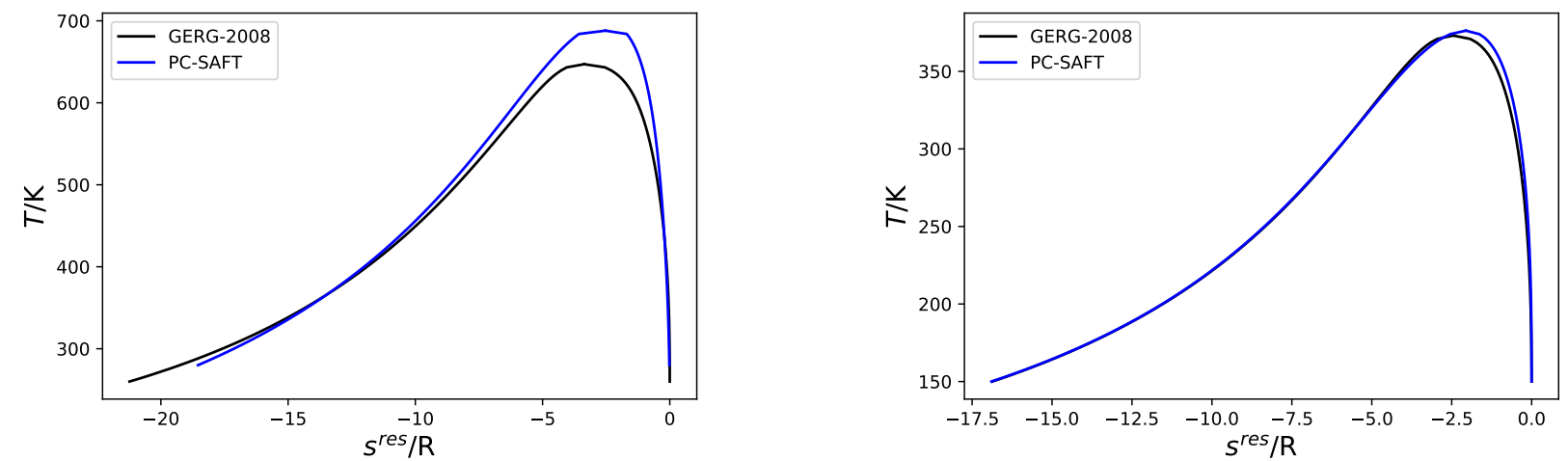

Figure S16: Residual entropy along the phase envelope for water (left) and $\mathrm{H}_{2} \mathrm{~S}$ (right) obtained from the pure-component EOS of GERG-2008 and PC-SAFT. PC-SAFT model parameters are taken from Refs. 28 and 34. 


\section{Model outputs for validation}

Tables S5 and S6 present model outputs for the 21 pure components and the resulting 210 binary pairs. These values can be used to validate the correct implementation of the presented viscosity model.

Table S5: Temperature $T$, pressure $p$, density $\rho(T, p)$, residual entropy $s^{r e s}(T, \rho)$, reference viscosity $\eta^{r e f}$ and calculated viscosity $\eta^{\text {model }}$ for the 21 components studied in this work.

\begin{tabular}{l|c|c|c|c|c|c} 
& $\mathrm{T} / \mathrm{K}$ & $\mathrm{p} / \mathrm{Pa}$ & $\rho / \mathrm{mol} / \mathrm{dm}^{3}$ & $s^{\text {res }} / \mathrm{R}$ & $\eta^{\text {ref }} /(\mathrm{Pa} \cdot \mathrm{s})$ & $\eta^{\text {model }} /(\mathrm{Pa} \cdot \mathrm{s})$ \\
\hline methane & 280.00 & 18511000 & 10.3563402 & -0.7635171 & $1.05905 \mathrm{E}-05$ & $1.89449 \mathrm{E}-05$ \\
ethane & 290.00 & 3514900 & 11.6836907 & -1.8544138 & $9.45289 \mathrm{E}-06$ & $4.37398 \mathrm{E}-05$ \\
propane & 300.00 & 8061200 & 11.5347181 & -3.1755625 & $8.70792 \mathrm{E}-06$ & $1.03519 \mathrm{E}-04$ \\
n-butane & 348.13 & 677520 & 0.2694947 & -0.1703608 & $9.20105 \mathrm{E}-06$ & $8.63244 \mathrm{E}-06$ \\
isobutane & 250.00 & 21018000 & 10.8064118 & -5.0564362 & $6.62835 \mathrm{E}-06$ & $3.25659 \mathrm{E}-04$ \\
n-pentane & 382.80 & 2000000 & 7.3335412 & -3.4863374 & $9.39688 \mathrm{E}-06$ & $1.08499 \mathrm{E}-04$ \\
isopentane & 498.15 & 980665 & 0.2574811 & -0.1112419 & $1.24348 \mathrm{E}-05$ & $1.18935 \mathrm{E}-05$ \\
hexane & 511.65 & 3200000 & 2.4648989 & -1.4912510 & $1.17452 \mathrm{E}-05$ & $2.98833 \mathrm{E}-05$ \\
heptane & 433.00 & 454000 & 5.4857254 & -4.0540629 & $9.38202 \mathrm{E}-06$ & $1.21764 \mathrm{E}-04$ \\
octane & 573.15 & 3044000 & 3.0150913 & -2.2749643 & $1.19145 \mathrm{E}-05$ & $4.41251 \mathrm{E}-05$ \\
nonane & 343.15 & 100000 & 5.2880845 & -7.4005831 & $6.74279 \mathrm{E}-06$ & $3.95292 \mathrm{E}-04$ \\
decane & 373.70 & 960000 & 4.6959765 & -7.3986529 & $7.11560 \mathrm{E}-06$ & $3.61481 \mathrm{E}-04$ \\
$\mathrm{CO}$ & 223.15 & 1370000 & 0.7556271 & -0.0487351 & $1.36976 \mathrm{E}-05$ & $1.40190 \mathrm{E}-05$ \\
$\mathrm{CO}{ }_{2}$ & 260.01 & 5034000 & 23.0153146 & -3.0664002 & $1.37481 \mathrm{E}-05$ & $1.32625 \mathrm{E}-04$ \\
$\mathrm{H}_{2}$ & 294.91 & 10160000 & 3.9034268 & -0.0819098 & $7.43761 \mathrm{E}-06$ & $8.83429 \mathrm{E}-06$ \\
$\mathrm{O}_{2}$ & 130.00 & 2381800 & 28.3714401 & -2.2096637 & $9.70506 \mathrm{E}-06$ & $7.68803 \mathrm{E}-05$ \\
$\mathrm{~N}_{2}$ & 101.01 & 1370000 & 24.4972121 & -2.4991542 & $6.84733 \mathrm{E}-06$ & $7.04351 \mathrm{E}-05$ \\
$\mathrm{H}_{2} \mathrm{~S}$ & 423.15 & 1010000 & 0.2944935 & -0.0329544 & $1.71553 \mathrm{E}-05$ & $1.66322 \mathrm{E}-05$ \\
$\mathrm{H}_{2} \mathrm{O}$ & 304.83 & 10000000 & 55.4885284 & -6.9212374 & $9.85740 \mathrm{E}-06$ & $7.71661 \mathrm{E}-04$ \\
helium & 100.18 & 35790000 & 28.4711691 & -0.4436549 & $7.58809 \mathrm{E}-06$ & $1.24309 \mathrm{E}-05$ \\
argon & 107.70 & 36000000 & 34.6673812 & -3.1478650 & $8.97412 \mathrm{E}-06$ & $2.23249 \mathrm{E}-04$
\end{tabular}

Table S6: Temperature $T$, density $\rho(T, p)$, residual entropy $s^{r e s}(T, \rho)$, reference viscosity $\eta^{\text {ref }}$ and calculated viscosity $\eta^{\text {model }}$ for all binary sub-systems of the 21 components studied in this work. The pressure is always 1 bar and the mole fraction of the first component is always $0.4 \mathrm{~mol} / \mathrm{mol}$.

\begin{tabular}{c|c|c|c|c|c|c} 
& & $\mathrm{T} / \mathrm{K}$ & $\rho / \mathrm{mol} / \mathrm{dm}^{3}$ & $s^{\text {res }} / \mathrm{R}$ & $\eta^{\text {ref }} /(\mathrm{Pa} \cdot \mathrm{s})$ & $\eta^{\text {model }} /(\mathrm{Pa} \cdot \mathrm{s})$ \\
\hline methane & $\mathrm{N}_{2}$ & 228.6768 & 0.05271062 & $-0.3646926 \mathrm{E}-02$ & $0.1236939 \mathrm{E}-04$ & $0.1167155 \mathrm{E}-04$
\end{tabular}




\begin{tabular}{|c|c|c|c|c|c|c|}
\hline methane & $\mathrm{CO}_{2}$ & 364.9538 & 0.03300954 & $-0.2812577 \mathrm{E}-02$ & $0.1758836 \mathrm{E}-04$ & $0.1486114 \mathrm{E}-04$ \\
\hline methane & ethane & 366.3864 & 0.03290447 & $-0.3577302 \mathrm{E}-02$ & $0.1233674 \mathrm{E}-04$ & $0.1092425 \mathrm{E}-04$ \\
\hline methane & propane & 443.7900 & 0.02716210 & $-0.3960517 \mathrm{E}-02$ & $0.1362689 \mathrm{E}-04$ & $0.1170386 \mathrm{E}-04$ \\
\hline methane & butane & 510.1500 & 0.02362998 & $-0.4503999 \mathrm{E}-02$ & $0.1449043 \mathrm{E}-04$ & $0.1269760 \mathrm{E}-04$ \\
\hline methane & isobutane & 489.3804 & 0.02463649 & $-0.4563838 \mathrm{E}-02$ & $0.1404697 \mathrm{E}-04$ & $0.1257097 \mathrm{E}-04$ \\
\hline methane & pentane & 563.6400 & 0.02138775 & $-0.4196224 \mathrm{E}-02$ & $0.1502835 \mathrm{E}-04$ & $0.1295972 \mathrm{E}-04$ \\
\hline methane & isopentane & 552.4200 & 0.02182477 & $-0.4483562 \mathrm{E}-02$ & $0.1500119 \mathrm{E}-04$ & $0.1365071 \mathrm{E}-04$ \\
\hline methane & hexane & 609.3840 & 0.01978059 & $-0.4531977 \mathrm{E}-02$ & $0.1534536 \mathrm{E}-04$ & $0.1285957 \mathrm{E}-04$ \\
\hline methane & heptane & 648.1560 & 0.01860202 & $-0.5082093 \mathrm{E}-02$ & $0.1548139 \mathrm{E}-04$ & $0.1322879 \mathrm{E}-04$ \\
\hline methane & octane & 683.1840 & 0.01764826 & $-0.5514104 \mathrm{E}-02$ & $0.1572664 \mathrm{E}-04$ & $0.1156700 \mathrm{E}-04$ \\
\hline methane & nonane & 713.4600 & 0.01690536 & $-0.6052110 \mathrm{E}-02$ & $0.1570574 \mathrm{E}-04$ & $0.1198314 \mathrm{E}-04$ \\
\hline methane & decane & 741.2400 & 0.01627377 & $-0.6485213 \mathrm{E}-02$ & $0.1581535 \mathrm{E}-04$ & $0.1451019 \mathrm{E}-04$ \\
\hline methane & $\mathrm{H}_{2}$ & 228.6768 & 0.05262089 & $-0.2042032 \mathrm{E}-02$ & $0.8572591 \mathrm{E}-05$ & $0.9138265 \mathrm{E}-05$ \\
\hline methane & $\mathrm{O}_{2}$ & 228.6768 & 0.05272895 & $-0.3399075 \mathrm{E}-02$ & $0.1364408 \mathrm{E}-04$ & $0.1256309 \mathrm{E}-04$ \\
\hline methane & $\mathrm{CO}$ & 228.6768 & 0.05272328 & $-0.3725378 \mathrm{E}-02$ & $0.1207515 \mathrm{E}-04$ & $0.1189154 \mathrm{E}-04$ \\
\hline methane & water & 776.5152 & 0.01549299 & $-0.7758929 \mathrm{E}-03$ & $0.2438977 \mathrm{E}-04$ & $0.2470676 \mathrm{E}-04$ \\
\hline methane & $\mathrm{H}_{2} \mathrm{~S}$ & 447.7200 & 0.02689301 & $-0.2084131 \mathrm{E}-02$ & $0.1746860 \mathrm{E}-04$ & $0.1668035 \mathrm{E}-04$ \\
\hline methane & he & 228.6768 & 0.05261698 & $-0.1405623 \mathrm{E}-02$ & $0.1061967 \mathrm{E}-04$ & $0.1222220 \mathrm{E}-04$ \\
\hline methane & argon & 228.6768 & 0.05273337 & $-0.3393396 \mathrm{E}-02$ & $0.1507402 \mathrm{E}-04$ & $0.1435351 \mathrm{E}-04$ \\
\hline $\mathrm{N}_{2}$ & $\mathrm{CO}_{2}$ & 364.9538 & 0.03299588 & $-0.2741876 \mathrm{E}-02$ & $0.1972443 \mathrm{E}-04$ & $0.1634170 \mathrm{E}-04$ \\
\hline $\mathrm{N}_{2}$ & ethane & 366.3864 & 0.03288306 & $-0.3319286 \mathrm{E}-02$ & $0.1461173 \mathrm{E}-04$ & $0.1268534 \mathrm{E}-04$ \\
\hline $\mathrm{N}_{2}$ & propane & 443.7900 & 0.02714548 & $-0.3350580 \mathrm{E}-02$ & $0.1563441 \mathrm{E}-04$ & $0.1316111 \mathrm{E}-04$ \\
\hline $\mathrm{N}_{2}$ & butane & 510.1500 & 0.02361564 & $-0.3659751 \mathrm{E}-02$ & $0.1631255 \mathrm{E}-04$ & $0.1400854 \mathrm{E}-04$ \\
\hline $\mathrm{N}_{2}$ & isobutane & 489.3804 & 0.02461997 & $-0.3735715 \mathrm{E}-02$ & $0.1582313 \mathrm{E}-04$ & $0.1387768 \mathrm{E}-04$ \\
\hline $\mathrm{N}_{2}$ & pentane & 563.6400 & 0.02137697 & $-0.3869053 \mathrm{E}-02$ & $0.1670691 \mathrm{E}-04$ & $0.1412497 \mathrm{E}-04$ \\
\hline $\mathrm{N}_{2}$ & isopentane & 552.4200 & 0.02181191 & $-0.3939852 \mathrm{E}-02$ & $0.1667034 \mathrm{E}-04$ & $0.1486992 \mathrm{E}-04$ \\
\hline $\mathrm{N}_{2}$ & hexane & 609.3840 & 0.01977172 & $-0.4409461 \mathrm{E}-02$ & $0.1690954 \mathrm{E}-04$ & $0.1389569 \mathrm{E}-04$ \\
\hline
\end{tabular}




\begin{tabular}{|c|c|c|c|c|c|c|}
\hline $\mathrm{N}_{2}$ & heptane & 648.1560 & 0.01859388 & $-0.5087551 \mathrm{E}-02$ & $0.1694942 \mathrm{E}-04$ & $0.1420466 \mathrm{E}-04$ \\
\hline $\mathrm{N}_{2}$ & octane & 683.1840 & 0.01764338 & $-0.5395351 \mathrm{E}-02$ & $0.1712421 \mathrm{E}-04$ & $0.1235168 \mathrm{E}-04$ \\
\hline $\mathrm{N}_{2}$ & nonane & 713.4600 & 0.01689525 & $-0.5540626 \mathrm{E}-02$ & $0.1703383 \mathrm{E}-04$ & $0.1274139 \mathrm{E}-04$ \\
\hline $\mathrm{N}_{2}$ & decane & 741.2400 & 0.01626338 & $-0.5738418 \mathrm{E}-02$ & 0.1709110E-04 & $0.1537138 \mathrm{E}-04$ \\
\hline $\mathrm{N}_{2}$ & $\mathrm{H}_{2}$ & 151.4304 & 0.07949708 & $-0.2943412 \mathrm{E}-02$ & $0.9277665 \mathrm{E}-05$ & $0.9703337 \mathrm{E}-05$ \\
\hline $\mathrm{N}_{2}$ & $\mathrm{O}_{2}$ & 185.5140 & 0.06505602 & $-0.4272282 \mathrm{E}-02$ & $0.1308783 \mathrm{E}-04$ & $0.1182728 \mathrm{E}-04$ \\
\hline $\mathrm{N}_{2}$ & $\mathrm{CO}$ & 159.4320 & 0.07583215 & $-0.6033821 \mathrm{E}-02$ & $0.1036970 \mathrm{E}-04$ & $0.1003203 \mathrm{E}-04$ \\
\hline $\mathrm{N}_{2}$ & water & 776.5152 & 0.01549123 & $-0.6677638 \mathrm{E}-03$ & $0.2943148 \mathrm{E}-04$ & $0.2922943 \mathrm{E}-04$ \\
\hline $\mathrm{N}_{2}$ & $\mathrm{H}_{2} \mathrm{~S}$ & 447.7200 & 0.02688362 & $-0.2015087 \mathrm{E}-02$ & $0.2009521 \mathrm{E}-04$ & $0.1881412 \mathrm{E}-04$ \\
\hline $\mathrm{N}_{2}$ & he & 151.4304 & 0.07940988 & $-0.2030113 \mathrm{E}-02$ & $0.1076105 \mathrm{E}-04$ & $0.1214687 \mathrm{E}-04$ \\
\hline $\mathrm{N}_{2}$ & argon & 180.8244 & 0.06675636 & $-0.4355151 \mathrm{E}-02$ & $0.1381499 \mathrm{E}-04$ & $0.1291167 \mathrm{E}-04$ \\
\hline $\mathrm{CO}_{2}$ & ethane & 366.3864 & 0.03292482 & $-0.4346436 \mathrm{E}-02$ & $0.1468278 \mathrm{E}-04$ & $0.1185943 \mathrm{E}-04$ \\
\hline $\mathrm{CO}_{2}$ & propane & 443.7900 & 0.02717131 & $-0.4203041 \mathrm{E}-02$ & $0.1589598 \mathrm{E}-04$ & $0.1244730 \mathrm{E}-04$ \\
\hline $\mathrm{CO}_{2}$ & butane & 510.1500 & 0.02363495 & $-0.4615337 \mathrm{E}-02$ & $0.1667384 \mathrm{E}-04$ & $0.1331954 \mathrm{E}-04$ \\
\hline $\mathrm{CO}_{2}$ & isobutane & 489.3804 & 0.02464166 & $-0.4618066 \mathrm{E}-02$ & $0.1614409 \mathrm{E}-04$ & $0.1317023 \mathrm{E}-04$ \\
\hline $\mathrm{CO}_{2}$ & pentane & 563.6400 & 0.02139132 & $-0.4641157 \mathrm{E}-02$ & $0.1711839 \mathrm{E}-04$ & $0.1346120 \mathrm{E}-04$ \\
\hline $\mathrm{CO}_{2}$ & isopentane & 552.4200 & 0.02182707 & $-0.4693990 \mathrm{E}-02$ & $0.1705333 \mathrm{E}-04$ & $0.1414786 \mathrm{E}-04$ \\
\hline $\mathrm{CO}_{2}$ & hexane & 609.3840 & 0.01978040 & $-0.4691088 \mathrm{E}-02$ & $0.1735250 \mathrm{E}-04$ & $0.1325699 \mathrm{E}-04$ \\
\hline $\mathrm{CO}_{2}$ & heptane & 648.1560 & 0.01860406 & $-0.5673610 \mathrm{E}-02$ & $0.1741252 \mathrm{E}-04$ & $0.1356999 \mathrm{E}-04$ \\
\hline $\mathrm{CO}_{2}$ & octane & 683.1840 & 0.01764823 & $-0.5975160 \mathrm{E}-02$ & $0.1759595 \mathrm{E}-04$ & $0.1180258 \mathrm{E}-04$ \\
\hline $\mathrm{CO}_{2}$ & nonane & 713.4600 & 0.01690272 & $-0.6187616 \mathrm{E}-02$ & $0.1751506 \mathrm{E}-04$ & $0.1218404 \mathrm{E}-04$ \\
\hline $\mathrm{CO}_{2}$ & decane & 741.2400 & 0.01627301 & $-0.7035092 \mathrm{E}-02$ & $0.1757534 \mathrm{E}-04$ & $0.1470663 \mathrm{E}-04$ \\
\hline $\mathrm{CO}_{2}$ & $\mathrm{H}_{2}$ & 364.9538 & 0.03296067 & $-0.1392255 \mathrm{E}-02$ & $0.1806662 \mathrm{E}-04$ & $0.1754471 \mathrm{E}-04$ \\
\hline $\mathrm{CO}_{2}$ & $\mathrm{O}_{2}$ & 364.9538 & 0.03298109 & $-0.1945095 \mathrm{E}-02$ & $0.2173102 \mathrm{E}-04$ & $0.1821334 \mathrm{E}-04$ \\
\hline $\mathrm{CO}_{2}$ & $\mathrm{CO}$ & 364.9538 & 0.03297666 & $-0.2152684 \mathrm{E}-02$ & $0.1973474 \mathrm{E}-04$ & $0.1769368 \mathrm{E}-04$ \\
\hline $\mathrm{CO}_{2}$ & water & 776.5152 & 0.01549500 & $-0.9807868 \mathrm{E}-03$ & $0.3081034 \mathrm{E}-04$ & $0.2844643 \mathrm{E}-04$ \\
\hline $\mathrm{CO}_{2}$ & $\mathrm{H}_{2} \mathrm{~S}$ & 447.7200 & 0.02690455 & $-0.2515989 \mathrm{E}-02$ & $0.2005191 \mathrm{E}-04$ & $0.1745697 \mathrm{E}-04$ \\
\hline
\end{tabular}




\begin{tabular}{|c|c|c|c|c|c|c|}
\hline $\mathrm{CO}_{2}$ & he & 364.9538 & 0.03295547 & $-0.9094060 \mathrm{E}-03$ & $0.2033020 \mathrm{E}-04$ & $0.2131922 \mathrm{E}-04$ \\
\hline $\mathrm{CO}_{2}$ & argon & 364.9538 & 0.03298323 & $-0.1965877 \mathrm{E}-02$ & $0.2322424 \mathrm{E}-04$ & $0.2012958 \mathrm{E}-04$ \\
\hline ethane & propane & 443.7900 & 0.02719293 & $-0.4895819 \mathrm{E}-02$ & $0.1320493 \mathrm{E}-04$ & $0.1067154 \mathrm{E}-04$ \\
\hline ethane & butane & 510.1500 & 0.02365000 & $-0.5098930 \mathrm{E}-02$ & $0.1415387 \mathrm{E}-04$ & $0.1166630 \mathrm{E}-04$ \\
\hline ethane & isobutane & 489.3804 & 0.02466043 & $-0.5334471 \mathrm{E}-02$ & $0.1370532 \mathrm{E}-04$ & $0.1153889 \mathrm{E}-04$ \\
\hline ethane & pentane & 563.6400 & 0.02140412 & $-0.5121727 \mathrm{E}-02$ & $0.1476174 \mathrm{E}-04$ & $0.1197765 \mathrm{E}-04$ \\
\hline ethane & isopentane & 552.4200 & 0.02183760 & $-0.5050998 \mathrm{E}-02$ & $0.1471148 \mathrm{E}-04$ & $0.1259214 \mathrm{E}-04$ \\
\hline ethane & hexane & 609.3840 & 0.01979730 & $-0.5901195 \mathrm{E}-02$ & $0.1514313 \mathrm{E}-04$ & $0.1194550 \mathrm{E}-04$ \\
\hline ethane & heptane & 648.1560 & 0.01861386 & $-0.6046634 \mathrm{E}-02$ & $0.1533838 \mathrm{E}-04$ & $0.1233334 \mathrm{E}-04$ \\
\hline ethane & octane & 683.1840 & 0.01765896 & $-0.6570336 \mathrm{E}-02$ & $0.1562196 \mathrm{E}-04$ & $0.1081402 \mathrm{E}-04$ \\
\hline ethane & nonane & 713.4600 & 0.01690527 & $-0.6686957 \mathrm{E}-02$ & $0.1564806 \mathrm{E}-04$ & $0.1123292 \mathrm{E}-04$ \\
\hline ethane & decane & 741.2400 & 0.01628252 & $-0.7551198 \mathrm{E}-02$ & $0.1578855 \mathrm{E}-04$ & $0.1363339 \mathrm{E}-04$ \\
\hline ethane & $\mathrm{H}_{2}$ & 366.3864 & 0.03283665 & $-0.1647237 \mathrm{E}-02$ & $0.1207535 \mathrm{E}-04$ & $0.1209477 \mathrm{E}-04$ \\
\hline ethane & $\mathrm{O}_{2}$ & 366.3864 & 0.03285902 & $-0.2309200 \mathrm{E}-02$ & $0.1799092 \mathrm{E}-04$ & $0.1555530 \mathrm{E}-04$ \\
\hline ethane & $\mathrm{CO}$ & 366.3864 & 0.03286032 & $-0.2820968 \mathrm{E}-02$ & $0.1606913 \mathrm{E}-04$ & $0.1486534 \mathrm{E}-04$ \\
\hline ethane & water & 776.5152 & 0.01549687 & $-0.1195990 \mathrm{E}-02$ & $0.2375568 \mathrm{E}-04$ & $0.2262054 \mathrm{E}-04$ \\
\hline ethane & $\mathrm{H}_{2} \mathrm{~S}$ & 447.7200 & 0.02691290 & $-0.2913787 \mathrm{E}-02$ & $0.1643533 \mathrm{E}-04$ & $0.1476119 \mathrm{E}-04$ \\
\hline ethane & he & 366.3864 & 0.03281451 & $-0.1116130 \mathrm{E}-02$ & $0.1422715 \mathrm{E}-04$ & $0.1538623 \mathrm{E}-04$ \\
\hline ethane & argon & 366.3864 & 0.03285841 & $-0.2262087 \mathrm{E}-02$ & $0.1965529 \mathrm{E}-04$ & $0.1757360 \mathrm{E}-04$ \\
\hline propane & butane & 510.1500 & 0.02366920 & $-0.5849192 \mathrm{E}-02$ & $0.1374172 \mathrm{E}-04$ & $0.1110313 \mathrm{E}-04$ \\
\hline propane & isobutane & 489.3804 & 0.02468091 & $-0.6031377 \mathrm{E}-02$ & $0.1329362 \mathrm{E}-04$ & 0.1097091E-04 \\
\hline propane & pentane & 563.6400 & 0.02141954 & $-0.5992908 \mathrm{E}-02$ & $0.1439592 \mathrm{E}-04$ & $0.1145206 \mathrm{E}-04$ \\
\hline propane & isopentane & 552.4200 & 0.02185540 & $-0.5910595 \mathrm{E}-02$ & $0.1432903 \mathrm{E}-04$ & $0.1202431 \mathrm{E}-04$ \\
\hline propane & hexane & 609.3840 & 0.01980747 & $-0.6538168 \mathrm{E}-02$ & $0.1482150 \mathrm{E}-04$ & $0.1146010 \mathrm{E}-04$ \\
\hline propane & heptane & 648.1560 & 0.01862534 & $-0.6971507 \mathrm{E}-02$ & $0.1505933 \mathrm{E}-04$ & $0.1187184 \mathrm{E}-04$ \\
\hline propane & octane & 683.1840 & 0.01766854 & $-0.7495905 \mathrm{E}-02$ & $0.1536927 \mathrm{E}-04$ & $0.1043111 \mathrm{E}-04$ \\
\hline propane & nonane & 713.4600 & 0.01692720 & $-0.8324694 \mathrm{E}-02$ & $0.1543160 \mathrm{E}-04$ & $0.1086821 \mathrm{E}-04$ \\
\hline
\end{tabular}




\begin{tabular}{|c|c|c|c|c|c|c|}
\hline propane & decane & 741.2400 & 0.01629097 & $-0.8369228 \mathrm{E}-02$ & $0.1559519 \mathrm{E}-04$ & $0.1320053 \mathrm{E}-04$ \\
\hline propane & $\mathrm{H}_{2}$ & 443.7900 & 0.02711180 & $-0.1697127 \mathrm{E}-02$ & $0.1343249 \mathrm{E}-04$ & $0.1318083 \mathrm{E}-04$ \\
\hline propane & $\mathrm{O}_{2}$ & 443.7900 & 0.02711907 & $-0.2192253 \mathrm{E}-02$ & $0.1929152 \mathrm{E}-04$ & $0.1633851 \mathrm{E}-04$ \\
\hline propane & $\mathrm{CO}$ & 443.7900 & 0.02712342 & $-0.2621988 \mathrm{E}-02$ & $0.1740695 \mathrm{E}-04$ & $0.1577267 \mathrm{E}-04$ \\
\hline propane & water & 776.5152 & 0.01550060 & $-0.1600206 \mathrm{E}-02$ & $0.2291994 \mathrm{E}-04$ & $0.2138641 \mathrm{E}-04$ \\
\hline propane & $\mathrm{H}_{2} \mathrm{~S}$ & 447.7200 & 0.02693186 & $-0.3658557 \mathrm{E}-02$ & $0.1552417 \mathrm{E}-04$ & $0.1366810 \mathrm{E}-04$ \\
\hline propane & he & 443.7900 & 0.02708971 & $-0.1142202 \mathrm{E}-02$ & $0.1549035 \mathrm{E}-04$ & $0.1641180 \mathrm{E}-04$ \\
\hline propane & argon & 443.7900 & 0.02711874 & $-0.2150587 \mathrm{E}-02$ & $0.2090713 \mathrm{E}-04$ & $0.1831045 \mathrm{E}-04$ \\
\hline butane & isobutane & 510.1500 & 0.02368428 & $-0.6615784 \mathrm{E}-02$ & $0.1338767 \mathrm{E}-04$ & $0.1119815 \mathrm{E}-04$ \\
\hline butane & pentane & 563.6400 & 0.02143829 & $-0.7095723 \mathrm{E}-02$ & $0.1402313 \mathrm{E}-04$ & $0.1131204 \mathrm{E}-04$ \\
\hline butane & isopentane & 552.4200 & 0.02187546 & $-0.7037904 \mathrm{E}-02$ & $0.1394293 \mathrm{E}-04$ & $0.1186464 \mathrm{E}-04$ \\
\hline butane & hexane & 609.3840 & 0.01982228 & $-0.7606515 \mathrm{E}-02$ & $0.1448468 \mathrm{E}-04$ & $0.1135597 \mathrm{E}-04$ \\
\hline butane & heptane & 648.1560 & 0.01863673 & $-0.7871328 \mathrm{E}-02$ & $0.1475723 \mathrm{E}-04$ & $0.1179377 \mathrm{E}-04$ \\
\hline butane & octane & 683.1840 & 0.01767916 & $-0.8432819 \mathrm{E}-02$ & $0.1508817 \mathrm{E}-04$ & $0.1038166 \mathrm{E}-04$ \\
\hline butane & nonane & 713.4600 & 0.01692878 & $-0.8617641 \mathrm{E}-02$ & $0.1518027 \mathrm{E}-04$ & $0.1083212 \mathrm{E}-04$ \\
\hline butane & decane & 741.2400 & 0.01629902 & $-0.9297631 \mathrm{E}-02$ & $0.1536245 \mathrm{E}-04$ & $0.1318092 \mathrm{E}-04$ \\
\hline butane & $\mathrm{H}_{2}$ & 510.1500 & 0.02358541 & $-0.1864872 \mathrm{E}-02$ & $0.1432081 \mathrm{E}-04$ & $0.1424087 \mathrm{E}-04$ \\
\hline butane & $\mathrm{O}_{2}$ & 510.1500 & 0.02358664 & $-0.2159737 \mathrm{E}-02$ & $0.2013809 \mathrm{E}-04$ & $0.1728129 \mathrm{E}-04$ \\
\hline butane & $\mathrm{CO}$ & 510.1500 & 0.02358857 & $-0.2521989 \mathrm{E}-02$ & $0.1831401 \mathrm{E}-04$ & $0.1681313 \mathrm{E}-04$ \\
\hline butane & water & 776.5152 & 0.01550460 & $-0.2057910 \mathrm{E}-02$ & $0.2209935 \mathrm{E}-04$ & $0.2090070 \mathrm{E}-04$ \\
\hline butane & $\mathrm{H}_{2} \mathrm{~S}$ & 510.1500 & 0.02362711 & $-0.3511528 \mathrm{E}-02$ & $0.1660998 \mathrm{E}-04$ & $0.1481508 \mathrm{E}-04$ \\
\hline butane & he & 510.1500 & 0.02356506 & $-0.1164479 \mathrm{E}-02$ & $0.1630515 \mathrm{E}-04$ & $0.1750548 \mathrm{E}-04$ \\
\hline butane & argon & 510.1500 & 0.02359600 & $-0.2479144 \mathrm{E}-02$ & $0.2169141 \mathrm{E}-04$ & $0.1925517 \mathrm{E}-04$ \\
\hline isobutane & pentane & 563.6400 & 0.02143376 & $-0.6842339 \mathrm{E}-02$ & $0.1405339 \mathrm{E}-04$ & $0.1149381 \mathrm{E}-04$ \\
\hline isobutane & isopentane & 552.4200 & 0.02187156 & $-0.6835675 \mathrm{E}-02$ & $0.1397427 \mathrm{E}-04$ & $0.1205694 \mathrm{E}-04$ \\
\hline isobutane & hexane & 609.3840 & 0.01981745 & $-0.7289239 \mathrm{E}-02$ & $0.1450930 \mathrm{E}-04$ & $0.1153257 \mathrm{E}-04$ \\
\hline isobutane & heptane & 648.1560 & 0.01863226 & $-0.7597684 \mathrm{E}-02$ & $0.1477755 \mathrm{E}-04$ & $0.1197386 \mathrm{E}-04$ \\
\hline
\end{tabular}




\begin{tabular}{|c|c|c|c|c|c|c|}
\hline isobutane & octane & 683.1840 & 0.01767353 & $-0.8045340 \mathrm{E}-02$ & $0.1510533 \mathrm{E}-04$ & $0.1053649 \mathrm{E}-04$ \\
\hline isobutane & nonane & 713.4600 & 0.01692594 & $-0.8400082 \mathrm{E}-02$ & $0.1519478 \mathrm{E}-04$ & $0.1099332 \mathrm{E}-04$ \\
\hline isobutane & decane & 741.2400 & 0.01629168 & $-0.8844119 \mathrm{E}-02$ & $0.1537497 \mathrm{E}-04$ & $0.1337308 \mathrm{E}-04$ \\
\hline isobutane & $\mathrm{H}_{2}$ & 489.3804 & 0.02457873 & $-0.1778165 \mathrm{E}-02$ & $0.1389266 \mathrm{E}-04$ & $0.1400922 \mathrm{E}-04$ \\
\hline isobutane & $\mathrm{O}_{2}$ & 489.3804 & 0.02458975 & $-0.2271152 \mathrm{E}-02$ & $0.1953236 \mathrm{E}-04$ & $0.1699964 \mathrm{E}-04$ \\
\hline isobutane & $\mathrm{CO}$ & 489.3804 & 0.02459160 & $-0.2646694 \mathrm{E}-02$ & $0.1776968 \mathrm{E}-04$ & $0.1654502 \mathrm{E}-04$ \\
\hline isobutane & water & 776.5152 & 0.01550403 & $-0.2004330 \mathrm{E}-02$ & $0.2213483 \mathrm{E}-04$ & $0.2122888 \mathrm{E}-04$ \\
\hline isobutane & $\mathrm{H}_{2} \mathrm{~S}$ & 489.3804 & 0.02463618 & $-0.3724558 \mathrm{E}-02$ & $0.1605458 \mathrm{E}-04$ & $0.1452429 \mathrm{E}-04$ \\
\hline isobutane & he & 489.3804 & 0.02456494 & $-0.1215251 \mathrm{E}-02$ & $0.1582005 \mathrm{E}-04$ & $0.1722463 \mathrm{E}-04$ \\
\hline isobutane & argon & 489.3804 & 0.02458951 & $-0.2227481 \mathrm{E}-02$ & $0.2104101 \mathrm{E}-04$ & $0.1893697 \mathrm{E}-04$ \\
\hline pentane & isopentane & 563.6400 & 0.02145242 & $-0.7826881 \mathrm{E}-02$ & $0.1384432 \mathrm{E}-04$ & $0.1166671 \mathrm{E}-04$ \\
\hline pentane & hexane & 609.3840 & 0.01983746 & $-0.8658486 \mathrm{E}-02$ & $0.1417069 \mathrm{E}-04$ & $0.1100505 \mathrm{E}-04$ \\
\hline pentane & heptane & 648.1560 & 0.01865025 & $-0.8929173 \mathrm{E}-02$ & $0.1447109 \mathrm{E}-04$ & $0.1145588 \mathrm{E}-04$ \\
\hline pentane & octane & 683.1840 & 0.01769325 & $-0.9674951 \mathrm{E}-02$ & $0.1481852 \mathrm{E}-04$ & $0.1010214 \mathrm{E}-04$ \\
\hline pentane & nonane & 713.4600 & 0.01695317 & $-0.1043304 \mathrm{E}-01$ & $0.1493482 \mathrm{E}-04$ & $0.1056433 \mathrm{E}-04$ \\
\hline pentane & decane & 741.2400 & 0.01631014 & $-0.1040708 \mathrm{E}-01$ & $0.1513179 \mathrm{E}-04$ & $0.1286060 \mathrm{E}-04$ \\
\hline pentane & $\mathrm{H}_{2}$ & 563.6400 & 0.02133957 & $-0.1801388 \mathrm{E}-02$ & $0.1487050 \mathrm{E}-04$ & $0.1463113 \mathrm{E}-04$ \\
\hline pentane & $\mathrm{O}_{2}$ & 563.6400 & 0.02134584 & $-0.2194653 \mathrm{E}-02$ & $0.2061544 \mathrm{E}-04$ & $0.1750532 \mathrm{E}-04$ \\
\hline pentane & $\mathrm{CO}$ & 563.6400 & 0.02134831 & $-0.2605669 \mathrm{E}-02$ & $0.1886711 \mathrm{E}-04$ & $0.1713990 \mathrm{E}-04$ \\
\hline pentane & water & 776.5152 & 0.01550905 & $-0.2549364 \mathrm{E}-02$ & $0.2135738 \mathrm{E}-04$ & $0.1999248 \mathrm{E}-04$ \\
\hline pentane & $\mathrm{H}_{2} \mathrm{~S}$ & 563.6400 & 0.02138206 & $-0.3584002 \mathrm{E}-02$ & $0.1738900 \mathrm{E}-04$ & $0.1534830 \mathrm{E}-04$ \\
\hline pentane & he & 563.6400 & 0.02132791 & $-0.1216406 \mathrm{E}-02$ & $0.1678171 \mathrm{E}-04$ & $0.1782740 \mathrm{E}-04$ \\
\hline pentane & argon & 563.6400 & 0.02134572 & $-0.2153349 \mathrm{E}-02$ & $0.2209957 \mathrm{E}-04$ & $0.1940530 \mathrm{E}-04$ \\
\hline isopentane & hexane & 609.3840 & 0.01983456 & $-0.8400737 \mathrm{E}-02$ & $0.1427268 \mathrm{E}-04$ & $0.1148379 \mathrm{E}-04$ \\
\hline isopentane & heptane & 648.1560 & 0.01864766 & $-0.8678627 \mathrm{E}-02$ & $0.1456658 \mathrm{E}-04$ & $0.1194725 \mathrm{E}-04$ \\
\hline isopentane & octane & 683.1840 & 0.01768744 & $-0.9119692 \mathrm{E}-02$ & 0.1490923E-04 & $0.1052738 \mathrm{E}-04$ \\
\hline isopentane & nonane & 713.4600 & 0.01693912 & $-0.9449083 \mathrm{E}-02$ & $0.1502015 \mathrm{E}-04$ & $0.1100024 \mathrm{E}-04$ \\
\hline
\end{tabular}




\begin{tabular}{|c|c|c|c|c|c|c|}
\hline isopentane & decane & 741.2400 & 0.01630422 & $-0.9938626 \mathrm{E}-02$ & $0.1521316 \mathrm{E}-04$ & $0.1339369 \mathrm{E}-04$ \\
\hline isopentane & $\mathrm{H}_{2}$ & 552.4200 & 0.02177323 & $-0.1809204 \mathrm{E}-02$ & $0.1485799 \mathrm{E}-04$ & $0.1515025 \mathrm{E}-04$ \\
\hline isopentane & $\mathrm{O}_{2}$ & 552.4200 & 0.02178012 & $-0.2213199 \mathrm{E}-02$ & $0.2051118 \mathrm{E}-04$ & $0.1805008 \mathrm{E}-04$ \\
\hline isopentane & $\mathrm{CO}$ & 552.4200 & 0.02178256 & $-0.2621192 \mathrm{E}-02$ & $0.1877580 \mathrm{E}-04$ & $0.1767699 \mathrm{E}-04$ \\
\hline isopentane & water & 776.5152 & 0.01550838 & $-0.2461064 \mathrm{E}-02$ & $0.2160292 \mathrm{E}-04$ & $0.2095606 \mathrm{E}-04$ \\
\hline isopentane & $\mathrm{H}_{2} \mathrm{~S}$ & 552.4200 & 0.02181459 & $-0.3251600 \mathrm{E}-02$ & $0.1726520 \mathrm{E}-04$ & $0.1578814 \mathrm{E}-04$ \\
\hline isopentane & he & 552.4200 & 0.02176114 & $-0.1226264 \mathrm{E}-02$ & $0.1675962 \mathrm{E}-04$ & $0.1845124 \mathrm{E}-04$ \\
\hline isopentane & argon & 552.4200 & 0.02177999 & $-0.2171529 \mathrm{E}-02$ & $0.2198031 \mathrm{E}-04$ & $0.2000237 \mathrm{E}-04$ \\
\hline hexane & heptane & 648.1560 & 0.01866148 & $-0.1036229 \mathrm{E}-01$ & $0.1418136 \mathrm{E}-04$ & $0.1102528 \mathrm{E}-04$ \\
\hline hexane & octane & 683.1840 & 0.01769952 & $-0.1076923 \mathrm{E}-01$ & $0.1454304 \mathrm{E}-04$ & $0.9734157 \mathrm{E}-05$ \\
\hline hexane & nonane & 713.4600 & 0.01695757 & $-0.1156217 \mathrm{E}-01$ & $0.1468065 \mathrm{E}-04$ & $0.1019607 \mathrm{E}-04$ \\
\hline hexane & decane & 741.2400 & 0.01631724 & $-0.1176404 \mathrm{E}-01$ & $0.1489056 \mathrm{E}-04$ & $0.1242661 \mathrm{E}-04$ \\
\hline hexane & $\mathrm{H}_{2}$ & 609.3840 & 0.01974644 & $-0.2071072 \mathrm{E}-02$ & $0.1519001 \mathrm{E}-04$ & $0.1466318 \mathrm{E}-04$ \\
\hline hexane & $\mathrm{O}_{2}$ & 609.3840 & 0.01974061 & $-0.2242089 \mathrm{E}-02$ & $0.2086320 \mathrm{E}-04$ & $0.1737866 \mathrm{E}-04$ \\
\hline hexane & $\mathrm{CO}$ & 609.3840 & 0.01974319 & $-0.2709179 \mathrm{E}-02$ & $0.1919287 \mathrm{E}-04$ & $0.1710462 \mathrm{E}-04$ \\
\hline hexane & water & 776.5152 & 0.01551275 & $-0.3143848 \mathrm{E}-02$ & $0.2062888 \mathrm{E}-04$ & $0.1894917 \mathrm{E}-04$ \\
\hline hexane & $\mathrm{H}_{2} \mathrm{~S}$ & 609.3840 & 0.01977395 & $-0.3901488 \mathrm{E}-02$ & $0.1789688 \mathrm{E}-04$ & 0.1549939E-04 \\
\hline hexane & he & 609.3840 & 0.01972609 & $-0.1240865 \mathrm{E}-02$ & $0.1703003 \mathrm{E}-04$ & $0.1774659 \mathrm{E}-04$ \\
\hline hexane & argon & 609.3840 & 0.01974055 & $-0.2198509 \mathrm{E}-02$ & $0.2228179 \mathrm{E}-04$ & $0.1919304 \mathrm{E}-04$ \\
\hline heptane & octane & 683.1840 & 0.01771362 & $-0.1207996 \mathrm{E}-01$ & $0.1426318 \mathrm{E}-04$ & $0.9678950 \mathrm{E}-05$ \\
\hline heptane & nonane & 713.4600 & 0.01696299 & $-0.1226516 \mathrm{E}-01$ & $0.1441980 \mathrm{E}-04$ & $0.1014793 \mathrm{E}-04$ \\
\hline heptane & decane & 741.2400 & 0.01632624 & $-0.1278212 \mathrm{E}-01$ & $0.1464123 \mathrm{E}-04$ & $0.1238285 \mathrm{E}-04$ \\
\hline heptane & $\mathrm{H}_{2}$ & 648.1560 & 0.01856583 & $-0.2096310 \mathrm{E}-02$ & $0.1532415 \mathrm{E}-04$ & $0.1498257 \mathrm{E}-04$ \\
\hline heptane & $\mathrm{O}_{2}$ & 648.1560 & 0.01855872 & $-0.2315262 \mathrm{E}-02$ & $0.2091862 \mathrm{E}-04$ & $0.1764934 \mathrm{E}-04$ \\
\hline heptane & $\mathrm{CO}$ & 648.1560 & 0.01856163 & $-0.2842381 \mathrm{E}-02$ & $0.1932729 \mathrm{E}-04$ & $0.1744674 \mathrm{E}-04$ \\
\hline heptane & water & 776.5152 & 0.01551782 & $-0.3741130 \mathrm{E}-02$ & $0.1991488 \mathrm{E}-04$ & $0.1853410 \mathrm{E}-04$ \\
\hline heptane & $\mathrm{H}_{2} \mathrm{~S}$ & 648.1560 & 0.01858898 & $-0.3713766 \mathrm{E}-02$ & $0.1818345 \mathrm{E}-04$ & $0.1594644 \mathrm{E}-04$ \\
\hline
\end{tabular}




\begin{tabular}{|c|c|c|c|c|c|c|}
\hline heptane & he & 648.1560 & 0.01854554 & $-0.1298677 \mathrm{E}-02$ & $0.1709250 \mathrm{E}-04$ & $0.1804074 \mathrm{E}-04$ \\
\hline heptane & argon & 648.1560 & 0.01855869 & $-0.2269640 \mathrm{E}-02$ & $0.2227504 \mathrm{E}-04$ & $0.1943444 \mathrm{E}-04$ \\
\hline octane & nonane & 713.4600 & 0.01697328 & $-0.1371357 \mathrm{E}-01$ & $0.1423809 \mathrm{E}-04$ & $0.9078337 \mathrm{E}-05$ \\
\hline octane & decane & 741.2400 & 0.01633682 & $-0.1434041 \mathrm{E}-01$ & $0.1446758 \mathrm{E}-04$ & $0.1108540 \mathrm{E}-04$ \\
\hline octane & $\mathrm{H}_{2}$ & 683.1840 & 0.01760295 & $-0.2134597 \mathrm{E}-02$ & $0.1557086 \mathrm{E}-04$ & $0.1377135 \mathrm{E}-04$ \\
\hline octane & $\mathrm{O}_{2}$ & 683.1840 & 0.01760524 & $-0.2404555 \mathrm{E}-02$ & $0.2109442 \mathrm{E}-04$ & $0.1610071 \mathrm{E}-04$ \\
\hline octane & $\mathrm{CO}$ & 683.1840 & 0.01760815 & $-0.2993029 \mathrm{E}-02$ & $0.1956229 \mathrm{E}-04$ & $0.1597587 \mathrm{E}-04$ \\
\hline octane & water & 776.5152 & 0.01552194 & $-0.4391063 \mathrm{E}-02$ & $0.1942998 \mathrm{E}-04$ & $0.1636459 \mathrm{E}-04$ \\
\hline octane & $\mathrm{H}_{2} \mathrm{~S}$ & 683.1840 & 0.01763188 & $-0.3723738 \mathrm{E}-02$ & $0.1852661 \mathrm{E}-04$ & $0.1469770 \mathrm{E}-04$ \\
\hline octane & he & 683.1840 & 0.01759363 & $-0.1356782 \mathrm{E}-02$ & $0.1728845 \mathrm{E}-04$ & $0.1650630 \mathrm{E}-04$ \\
\hline octane & argon & 683.1840 & 0.01760524 & $-0.2357246 \mathrm{E}-02$ & $0.2240111 \mathrm{E}-04$ & $0.1768089 \mathrm{E}-04$ \\
\hline nonane & decane & 741.2400 & 0.01634915 & $-0.1554754 \mathrm{E}-01$ & $0.1422895 \mathrm{E}-04$ & $0.1117994 \mathrm{E}-04$ \\
\hline nonane & $\mathrm{H}_{2}$ & 713.4600 & 0.01685584 & $-0.2301553 \mathrm{E}-02$ & $0.1554537 \mathrm{E}-04$ & $0.1408643 \mathrm{E}-04$ \\
\hline nonane & $\mathrm{O}_{2}$ & 713.4600 & 0.01685756 & $-0.2524012 \mathrm{E}-02$ & $0.2099095 \mathrm{E}-04$ & $0.1641516 \mathrm{E}-04$ \\
\hline nonane & $\mathrm{CO}$ & 713.4600 & 0.01686077 & $-0.3182245 \mathrm{E}-02$ & $0.1952823 \mathrm{E}-04$ & $0.1634020 \mathrm{E}-04$ \\
\hline nonane & water & 776.5152 & 0.01552777 & $-0.5046808 \mathrm{E}-02$ & $0.1879712 \mathrm{E}-04$ & $0.1622520 \mathrm{E}-04$ \\
\hline nonane & $\mathrm{H}_{2} \mathrm{~S}$ & 713.4600 & 0.01688833 & $-0.4153898 \mathrm{E}-02$ & $0.1860270 \mathrm{E}-04$ & $0.1512446 \mathrm{E}-04$ \\
\hline nonane & he & 713.4600 & 0.01684623 & $-0.1453075 \mathrm{E}-02$ & $0.1719911 \mathrm{E}-04$ & $0.1682308 \mathrm{E}-04$ \\
\hline nonane & argon & 713.4600 & 0.01685757 & $-0.2474669 \mathrm{E}-02$ & $0.2224652 \mathrm{E}-04$ & $0.1799002 \mathrm{E}-04$ \\
\hline decane & $\mathrm{H}_{2}$ & 741.2400 & 0.01623809 & $-0.2422455 \mathrm{E}-02$ & $0.1565406 \mathrm{E}-04$ & $0.1604255 \mathrm{E}-04$ \\
\hline decane & $\mathrm{O}_{2}$ & 741.2400 & 0.01622513 & $-0.2547258 \mathrm{E}-02$ & $0.2103110 \mathrm{E}-04$ & $0.1859910 \mathrm{E}-04$ \\
\hline decane & $\mathrm{CO}$ & 741.2400 & 0.01623827 & $-0.2989684 \mathrm{E}-02$ & $0.1962052 \mathrm{E}-04$ & $0.1856244 \mathrm{E}-04$ \\
\hline decane & water & 776.5152 & 0.01553302 & $-0.5792921 \mathrm{E}-02$ & $0.1836549 \mathrm{E}-04$ & $0.1793117 \mathrm{E}-04$ \\
\hline decane & $\mathrm{H}_{2} \mathrm{~S}$ & 741.2400 & 0.01625580 & $-0.4428019 \mathrm{E}-02$ & $0.1877463 \mathrm{E}-04$ & $0.1726342 \mathrm{E}-04$ \\
\hline decane & he & 741.2400 & 0.01621489 & $-0.1455242 \mathrm{E}-02$ & $0.1726175 \mathrm{E}-04$ & $0.1909531 \mathrm{E}-04$ \\
\hline decane & argon & 741.2400 & 0.01622516 & $-0.2495454 \mathrm{E}-02$ & $0.2224570 \mathrm{E}-04$ & $0.2034385 \mathrm{E}-04$ \\
\hline $\mathrm{H}_{2}$ & $\mathrm{O}_{2}$ & 185.5140 & 0.06490455 & $-0.2567572 \mathrm{E}-02$ & $0.1307213 \mathrm{E}-04$ & $0.1280377 \mathrm{E}-04$ \\
\hline
\end{tabular}




\begin{tabular}{|c|c|c|c|c|c|c|}
\hline $\mathrm{H}_{2}$ & $\mathrm{CO}$ & 159.4320 & 0.07563034 & $-0.3985400 \mathrm{E}-02$ & $0.9902579 \mathrm{E}-05$ & $0.1038173 \mathrm{E}-04$ \\
\hline $\mathrm{H}_{2}$ & water & 776.5152 & 0.01548940 & $-0.4110672 \mathrm{E}-03$ & $0.2420841 \mathrm{E}-04$ & $0.2610459 \mathrm{E}-04$ \\
\hline $\mathrm{H}_{2}$ & $\mathrm{H}_{2} \mathrm{~S}$ & 447.7200 & 0.02686854 & $-0.1246345 \mathrm{E}-02$ & $0.1797106 \mathrm{E}-04$ & $0.1825702 \mathrm{E}-04$ \\
\hline $\mathrm{H}_{2}$ & he & 39.82800 & 0.3027363 & $-0.6175059 \mathrm{E}-02$ & $0.3127522 \mathrm{E}-05$ & $0.3837486 \mathrm{E}-05$ \\
\hline $\mathrm{H}_{2}$ & argon & 180.8244 & 0.06659391 & $-0.2601344 \mathrm{E}-02$ & $0.1429325 \mathrm{E}-04$ & $0.1447808 \mathrm{E}-04$ \\
\hline $\mathrm{O}_{2}$ & $\mathrm{CO}$ & 185.5140 & 0.06506626 & $-0.4435290 \mathrm{E}-02$ & $0.1248872 \mathrm{E}-04$ & $0.1187410 \mathrm{E}-04$ \\
\hline $\mathrm{O}_{2}$ & water & 776.5152 & 0.01549172 & $-0.6371414 \mathrm{E}-03$ & $0.3209071 \mathrm{E}-04$ & $0.3135666 \mathrm{E}-04$ \\
\hline $\mathrm{O}_{2}$ & $\mathrm{H}_{2} \mathrm{~S}$ & 447.7200 & 0.02688578 & $-0.1756388 \mathrm{E}-02$ & $0.2137046 \mathrm{E}-04$ & $0.1968194 \mathrm{E}-04$ \\
\hline $\mathrm{O}_{2}$ & he & 185.5140 & 0.06481167 & $-0.1536215 \mathrm{E}-02$ & $0.1390073 \mathrm{E}-04$ & $0.1543508 \mathrm{E}-04$ \\
\hline $\mathrm{O}_{2}$ & argon & 185.5140 & 0.06507192 & $-0.3999468 \mathrm{E}-02$ & 0.1469391E-04 & $0.1350840 \mathrm{E}-04$ \\
\hline $\mathrm{CO}$ & water & 776.5152 & 0.01549148 & $-0.6880271 \mathrm{E}-03$ & $0.2905817 \mathrm{E}-04$ & 0.2970103E-04 \\
\hline $\mathrm{CO}$ & $\mathrm{H}_{2} \mathrm{~S}$ & 447.7200 & 0.02688536 & $-0.1908798 \mathrm{E}-02$ & $0.1987428 \mathrm{E}-04$ & $0.1914606 \mathrm{E}-04$ \\
\hline $\mathrm{CO}$ & he & 159.4320 & 0.07540969 & $-0.2018027 \mathrm{E}-02$ & $0.1091467 \mathrm{E}-04$ & $0.1267798 \mathrm{E}-04$ \\
\hline $\mathrm{CO}$ & argon & 180.8244 & 0.06678135 & $-0.4674776 \mathrm{E}-02$ & $0.1361669 \mathrm{E}-04$ & $0.1309636 \mathrm{E}-04$ \\
\hline water & $\mathrm{H}_{2} \mathrm{~S}$ & 776.5152 & 0.01549494 & $-0.9957420 \mathrm{E}-03$ & $0.2790518 \mathrm{E}-04$ & $0.2741050 \mathrm{E}-04$ \\
\hline water & he & 776.5152 & 0.01548742 & $-0.2344484 \mathrm{E}-03$ & $0.2761536 \mathrm{E}-04$ & $0.3271124 \mathrm{E}-04$ \\
\hline water & argon & 776.5152 & 0.01548909 & $-0.5368776 \mathrm{E}-03$ & $0.3870497 \mathrm{E}-04$ & $0.3785340 \mathrm{E}-04$ \\
\hline $\mathrm{H}_{2} \mathrm{~S}$ & he & 447.7200 & 0.02685646 & $-0.7113955 \mathrm{E}-03$ & $0.2006405 \mathrm{E}-04$ & $0.2283524 \mathrm{E}-04$ \\
\hline $\mathrm{H}_{2} \mathrm{~S}$ & argon & 447.7200 & 0.02687491 & $-0.1443203 \mathrm{E}-02$ & $0.2523014 \mathrm{E}-04$ & $0.2372423 \mathrm{E}-04$ \\
\hline he & argon & 180.8244 & 0.06654689 & $-0.1996979 \mathrm{E}-02$ & $0.1512990 \mathrm{E}-04$ & $0.1612406 \mathrm{E}-04$ \\
\hline
\end{tabular}




\section{References}

(1) Atilhan, M.; Aparicio, S.; Alcalde, R.; Iglesias-Silva, G. A.; El-Halwagi, M.; Hall, K. R. Viscosity measurements and data correlation for two synthetic natural gas mixtures. Journal of Chemical \& Engineering Data 2010, 55, 2498-2504.

(2) Atilhan, M.; Aparicio, S.; Iglesias-Silva, G. A.; El-Halwagi, M.; Hall, K. R. On the viscosity of natural gases from Qatari north field reservoir. Journal of Chemical \& Engineering Data 2010, 55, 5117-5123.

(3) Assael, M.; Dalaouti, N.; Vesovic, V. Viscosity of natural-gas mixtures: measurements and prediction. International journal of thermophysics 2001, 22, 61-71.

(4) Langelandsvik, L.; Solvang, S.; Rousselet, M.; Metaxa, I.; Assael, M. Dynamic viscosity measurements of three natural gas mixtures - comparison against prediction models. International journal of thermophysics 2007, 28, 1120-1130.

(5) Schley, P.; Jaeschke, M.; Küchenmeister, C.; Vogel, E. Viscosity measurements and predictions for natural gas. International journal of thermophysics 2004, 25, 16231652.

(6) Lee, A. L.; Gonzalez, M. H.; Eakin, B. E., et al. The viscosity of natural gases. Journal of Petroleum Technology 1966, 18, 997-1.

(7) Kashefi, K.; Chapoy, A.; Bell, K.; Tohidi, B. Viscosity of binary and multicomponent hydrocarbon fluids at high pressure and high temperature conditions: Measurements and predictions. Journal of Petroleum Science and Engineering 2013, 112, 153-160.

(8) Elsharkawy, A. M. Efficient methods for calculations of compressibility, density and viscosity of natural gases. Fluid Phase Equilibria 2004, 218, 1-13. 
(9) Sanjari, E.; Lay, E. N.; Peymani, M. An accurate empirical correlation for predicting natural gas viscosity. Journal of natural gas chemistry 2011, 20, 654-658.

(10) Heidaryan, E.; Salarabadi, A.; Moghadasi, J. A novel correlation approach for prediction of natural gas compressibility factor. Journal of Natural Gas Chemistry 2010, 19, 189192.

(11) Carr, N. L.; Kobayashi, R.; Burrows, D. B., et al. Viscosity of hydrocarbon gases under pressure. Journal of Petroleum Technology 1954, 6, 47-55.

(12) Yarranton, H.; Satyro, M. Expanded fluid-based viscosity correlation for hydrocarbons. Industrial \&6 engineering chemistry research 2009, 48, 3640-3648.

(13) Motahhari, H.; Satyro, M.; Yarranton, H. Viscosity prediction for natural gas processing applications. Fluid phase equilibria 2012, 322, 56-65.

(14) Satyro, M.; Yarranton, H. Expanded fluid-based viscosity correlation for hydrocarbons using an equation of state. Fluid Phase Equilibria 2010, 298, 1-11.

(15) Huber, M. NIST thermophysical properties of hydrocarbon mixtures database (SUPERTRAPP). NIST Standard Reference Database 2003, 4.

(16) Chung, T. H.; Lee, L. L.; Starling, K. E. Applications of kinetic gas theories and multiparameter correlation for prediction of dilute gas viscosity and thermal conductivity. Industrial \& engineering chemistry fundamentals 1984, 23, 8-13.

(17) Chung, T. H.; Ajlan, M.; Lee, L. L.; Starling, K. E. Generalized multiparameter correlation for nonpolar and polar fluid transport properties. Industrial \& engineering chemistry research 1988, 27, 671-679.

(18) Lohrenz, J.; Bray, B. G.; Clark, C. R., et al. Calculating viscosities of reservoir fluids from their compositions. Journal of Petroleum Technology 1964, 16, 1-171. 
(19) Pedersen, K. S.; Fredenslund, A.; Christensen, P. L.; Thomassen, P. Viscosity of crude oils. Chemical Engineering Science 1984, 39, 1011-1016.

(20) Pedersen, K. S.; Fredenslund, A. An improved corresponding states model for the prediction of oil and gas viscosities and thermal conductivities. Chemical Engineering Science 1987, 42, 182-186.

(21) Novak, L. T. Predicting natural gas viscosity with a mixture viscosity model for the entire fluid region. Industrial \& Engineering Chemistry Research 2013, 52, 1601416018.

(22) Whitson, C. H.; Brule, M. R. Phase Behavior, Vol. 20. Richardson, Texas: Monograph Series, SPE 2000,

(23) Londono, F. E.; Archer, R. A.; Blasingame, T. A., et al. Correlations for hydrocarbon gas viscosity and gas density-validation and correlation of behavior using a large-scale database. SPE Reservoir Evaluation \&6 Engineering 2005, 8, 561-572.

(24) Poling, B. E.; Prausnitz, J. M.; O'connell, J. P., et al. The properties of gases and liquids; Mcgraw-hill New York, 2001; Vol. 5.

(25) Vesovic, V. Predicting the viscosity of natural gas. International journal of thermophysics 2001, 22, 415-426.

(26) Al-Syabi, Z.; Danesh, A.; Tohidi, B.; Todd, A. C.; Tehrani, D. A residual viscosity correlation for predicting the viscosity of petroleum reservoir fluids over wide ranges of pressure and temperature. Chemical engineering science 2001, 56, 6997-7006.

(27) Gross, J.; Sadowski, G. Perturbed-chain SAFT: An equation of state based on a perturbation theory for chain molecules. Industrial $\&$ engineering chemistry research 2001, 40, $1244-1260$. 
(28) Gross, J.; Sadowski, G. Application of the perturbed-chain SAFT equation of state to associating systems. Industrial \& engineering chemistry research 2002, 41, 5510-5515.

(29) Gross, J. An equation-of-state contribution for polar components: Quadrupolar molecules. AIChE journal 2005, 51, 2556-2568.

(30) Gross, J.; Vrabec, J. An equation-of-state contribution for polar components: Dipolar molecules. AIChE journal 2006, 52, 1194-1204.

(31) Vrabec, J.; Gross, J. Vapor-liquid equilibria simulation and an equation of state contribution for dipole-quadrupole interactions. The Journal of Physical Chemistry B 2008, 112, 51-60.

(32) Hirschfelder, J. O.; Curtiss, C. F.; Bird, R. B.; Mayer, M. G. Molecular theory of gases and liquids; Wiley New York, 1964; Vol. 165.

(33) Vins, V.; Vacek, V. Effect of gas impurities on the throttling process of fluorocarbon refrigerants: estimation of the Henry's law constant. Journal of Chemical \& Engineering Data 2009, 54, 2395-2403.

(34) Tang, X.; Gross, J. Modeling the phase equilibria of hydrogen sulfide and carbon dioxide in mixture with hydrocarbons and water using the PCP-SAFT equation of state. Fluid phase equilibria 2010, 293, 11-21. 